\title{
Insulin and IGF-1 receptors regulate complex I- dependent mitochondrial bioenergetics and supercomplexes via FoxOs in muscle
}

\author{
Gourav Bhardwaj, ${ }^{1,2}$ Christie M. Penniman, ${ }^{1,2}$ Jayashree Jena, ${ }^{1}$ Pablo A. Suarez Beltran, ${ }^{1}$ Collin Foster, ${ }^{1}$ Kennedy Poro, ${ }^{1}$ \\ Taylor L. Junck, ${ }^{1}$ Antentor O. Hinton Jr., ${ }^{1}$ Rhonda Souvenir, ${ }^{1}$ Jordan D. Fuqua, ${ }^{3}$ Pablo E. Morales, ${ }^{4}$ Roberto Bravo-Sagua, ${ }^{4}$ \\ William I. Sivitz, ${ }^{1,2}$ Vitor A. Lira, ${ }^{3}$ E. Dale Abel, ${ }^{1}$ and Brian T. O'Neill ${ }^{1,2}$
}

${ }^{1}$ Fraternal Order of Eagles Diabetes Research Center and Division of Endocrinology and Metabolism, Roy J. and Lucille A. Carver College of Medicine, University of lowa, lowa City, lowa, USA. ${ }^{2}$ Veterans Affairs Health Care System, lowa City, lowa, USA. ${ }^{3}$ Fraternal Order of Eagles Diabetes Research Center and Department of Health and Human Physiology, College of Liberal Arts and Sciences, University of lowa, Iowa City, lowa, USA. ${ }^{4}$ Advanced Center for Chronic Diseases (ACCDiS), University of Chile, Santiago, Chile.

\begin{abstract}
Decreased skeletal muscle strength and mitochondrial dysfunction are characteristic of diabetes. The actions of insulin and IGF-1 through the insulin receptor (IR) and IGF-1 receptor (IGF1R) maintain muscle mass via suppression of forkhead box $\mathrm{O}$ (FoxO) transcription factors, but whether FoxO activation coordinates atrophy in concert with mitochondrial dysfunction is unknown. We show that mitochondrial respiration and complex I activity were decreased in streptozotocin (STZ) diabetic muscle, but these defects were reversed in muscle-specific Fox01, -3, and -4 triple-KO (M-FoxO TKO) mice rendered diabetic with STZ. In the absence of systemic glucose or lipid abnormalities, muscle-specific IR KO (M-IR $\left.{ }^{-/-}\right)$or combined IR/IGF1R KO (MIGIRKO) impaired mitochondrial respiration, decreased ATP production, and increased ROS. These mitochondrial abnormalities were not present in muscle-specific IR, IGF1R, and Fox01, -3, and -4 quintuple-KO mice (M-QKO). Acute tamoxifen-inducible deletion of IR and IGF1R also decreased muscle pyruvate respiration, complex I activity, and supercomplex assembly. Although autophagy was increased when IR and IGF1R were deleted in muscle, mitophagy was not increased. Mechanistically, RNA-Seq revealed that complex I core subunits were decreased in STZ-diabetic and MICIRKO muscle, and these changes were not present with FoxO KO in STZ-FoxO TKO and M-QKO mice. Thus, insulin-deficient diabetes or loss of insulin/IGF-1 action in muscle decreases complex I-driven mitochondrial respiration and supercomplex assembly in part by FoxO-mediated repression of complex I subunit expression.
\end{abstract}

\section{Introduction}

Decreased muscle strength is associated with poorly controlled diabetes and contributes to physical disability, frailty, and increased risk for morbidity and mortality in older individuals. Muscle strength declines faster in older diabetic patients, leaving them at higher risk of death $(1,2)$. Both type 1 and type 2 diabetes patients show decreased muscle strength, muscle quality (strength per unit muscle mass), and overall fitness compared with nondiabetic individuals $(3,4)$. Mitochondrial abnormalities, including decreased respiratory capacity, decreased ATP synthesis, and increased ROS generation, have been reported in muscle of type 1 and type 2 diabetic patients (5-9). Yet other reports indicate that mitochondrial respiration and function are normal in muscle from patients with prediabetes or insulin resistance $(10,11)$. The mechanisms that control muscle mitochondrial function in diabetes and how this relates to muscle strength and physiology are poorly understood. Thus, the cause

Conflict of interest: The authors have declared that no conflict of interest exists. Copyright: @ 2021, American Society for Clinical Investigation.

Submitted: December 3, 2020; Accepted: July 28, 2021; Published: September 15, 2021 Reference information: / Clin Invest. 2021;131(18):e146415.

https://doi.org/10.1172/JCl146415. and effect relationship between mitochondrial dysfunction and diabetes remains controversial.

Insulin induces muscle glucose uptake and regulates protein turnover (12). In patients with type 1 diabetes, short-term withdrawal of insulin leads to a high protein-turnover state, which drives loss of muscle mass $(13,14)$. Past and recent studies in mice and humans indicate that loss of insulin action in diabetes decreases muscle mitochondrial respiration and suppresses the mitochondrial proteome, which may be prevented with insulin treatment, suggesting a beneficial effect of insulin on maintenance of mitochondrial function $(6,15)$. Alterations in mitochondrial structure are associated with these defects in mitochondrial function in patients with type 1 diabetes (9). These studies show that insulin action in muscle improves mitochondrial function, but the molecular targets that mediate these responses and the signaling abnormalities that alter mitochondrial function in diabetes are not known. Thus, delineating insulin-mediated pathways that regulate mitochondrial metabolism could provide an important target for improving muscle strength and preventing disability in patients with diabetes.

Insulin signals via insulin receptors (IRs) to regulate metabolic changes and cellular growth. IR and highly homologous IGF-1 receptors (IGF1R) share common downstream signaling path- 
ways that regulate muscle glucose uptake and protein turnover via IRS/PI3K/Akt $(16,17)$. Prior work indicates that loss of IR or IGF1R specifically in muscle leads to mild decrease in muscle size $(18,19)$, but combined loss of IR and IGF1R in muscle induces a marked decrease in muscle mass via activation of Forkhead box O-dependent (FoxO-dependent) autophagy and proteasomal degradation pathways, indicating that signaling via IR/IGF1R is critical for maintenance of muscle mass (20).

FoxO proteins are evolutionarily conserved metabolic and stress-responsive transcription factors that regulate genes related to autophagy, oxidative stress, energy metabolism, and muscle atrophy $(21,22)$. In cells and various tissues, FoxOs regulate mitochondrial metabolism $(23,24)$. In tumor cells, activation of FoxO3 suppresses mitochondrial gene expression, which modulates responses to hypoxia (25). Loss of IRS1 and IRS2 in liver decreases mitochondrial oxidative metabolism and alters fission and fusion proteins, and these mitochondrial defects were normalized by FoxO1 deletion in liver (26). In muscle, the regulation of mitochondrial metabolism by FoxOs is less clear. Constitutive activation of FoxO3 induces mitochondrial fission that is associated with muscle loss (27), but loss of FoxO1, -3, and -4 did not alter the balance of oxidative or glycolytic fibers $(28,29)$. Still other reports indicate that mitochondrial dysfunction from loss of Opa1 can activate FoxOs (30), possibly indicating a reciprocal relationship between mitochondria and FoxOs. Demonstration of direct regulation of mitochondrial metabolic function in muscle by insulin/FoxO signaling and how this may coordinate with regulation of muscle mass remain to be determined.

This study aims to determine the role of FoxOs in mitochondrial function in insulin deficiency and the relative roles of IR, IGF1R, or both IR/IGF1R and FoxOs in muscle mitochondrial metabolism. We show that in a model of type 1 diabetes, mitochondrial respiration, ATP production, and complex I activity were decreased in streptozotocin (STZ) diabetic muscle, and these mitochondrial abnormalities were reversed with the deletion of FoxOs in STZ-FoxO triple-KO (TKO) mice. Furthermore, we show that perinatal muscle-specific loss of IR $\left(\mathrm{M}-\mathrm{IR}^{-/-}\right)$ caused a mild decrease in pyruvate-driven respiration in soleus fibers, whereas loss of IGF1R in M-IGF1R ${ }^{-/-}$did not. Combined deletion of both IR and IGF1R (MIGIRKO) resulted in a dramatic decrease in respiration and ATP production with increased ROS. These mitochondrial abnormalities were not present when muscle-specific loss of FoxO1, -3 , and -4 was introduced onto the IR/IGF1R-deficient background (quintuple KO [M-QKO]), indicating that FoxO activation mediates mitochondrial decline. Tamoxifen-inducible IR/IGF1R KO in adult muscle (INDIGIRKO) demonstrated a decrease in soleus fiber respiration, mitochondrial content, and complex I expression and activity. FoxOs are known inducers of autophagy, but mitophagy quantification and flux were not increased with deletion of IR/IGF1R. Mechanistically, RNA-Seq demonstrates that complex I subunits were decreased in STZ-diabetic and MIGIRKO muscle, but were unchanged in M-QKO and STZ-FoxO TKO compared with control mice. Thus, loss of insulin action via IR or both IR/IGF1R receptors in muscle decreases complex I-driven mitochondrial respiration and supercomplex assembly, in part by FoxO-mediated repression of mitochondrial genes.

\section{Results}

Deletion of FoxOs in muscle prevents complex I-mediated mitochondrial defects in response to insulin-deficient diabetes. Insulin deficiency is known to decrease muscle mitochondrial bioenergetics in mice rendered diabetic with STZ (15). We have shown that FoxOs regulate loss of muscle mass after STZ diabetes in mice (28). The current study examines the role of FoxOs in the coordination of muscle atrophy and diabetes-induced alterations of muscle mitochondrial function. Muscle-specific FoxO1, -3, and -4 triple-KO (M-FoxO TKO) mice and FoxO1, -3 , and -4 triple-floxed $\left(\mathrm{T}^{\mathrm{f} / \mathrm{f}}\right)$ control mice were rendered diabetic with STZ, and mitochondrial functional and biochemical assessments were performed 14 days after high-dose STZ injection.

Maximal respiration in mitochondria isolated from 1 full quadriceps and 1 full gastrocnemius (quad/gast) muscle was decreased in STZ diabetic $\mathrm{T}^{\mathrm{f} / \mathrm{fl}}$ mice compared with citrate-treated $\mathrm{T}^{\mathrm{f} / \mathrm{fl}}$ controls when using glutamate/malate and succinate substrates (Figure 1A). ATP synthesis rates were also decreased in mitochondria from STZ diabetic $\mathrm{T}^{\mathrm{fl} / \mathrm{fl}}$ muscle under the same conditions (Figure 1B). Deletion of FoxOs rescued the mitochondrial defects in STZ muscle, which was demonstrated by the fact that maximal respiration and ATP production in STZ-FoxO TKO mitochondria were not different from those in either $\mathrm{T}^{\mathrm{f} / \mathrm{fl}}$ or $\mathrm{M}$-FoxO TKO mice (Figure $1, \mathrm{~A}$ and $\mathrm{B}) . \mathrm{H}_{2} \mathrm{O}_{2}$ production in isolated mitochondria was highly variable at low ADP levels, but was increased in STZ diabetic $\mathrm{T}^{\mathrm{f} / \mathrm{fl}}$ and STZ-FoxO TKO with $1000 \mu \mathrm{M}$ ADP (Figure 1C). Interestingly, although soleus muscle weight was increased in M-FoxO TKO mice (Supplemental Figure 1A; supplemental material available online with this article; https://oi.org/10.1172/JCI146415DS1), permeabilized soleus fibers showed no differences in respiratory capacity among the 4 groups (Supplemental Figure 1B).

We analyzed transcript levels of mitochondrial oxidative phosphorylation (OXPHOS) subunits from a prior RNA-Seq analysis on M-FoxO TKO and $\mathrm{T}^{\mathrm{fl} / \mathrm{fl}}$ control mice treated with STZ (28). Twenty-seven out of 92 OXPHOS genes (Figure 1D and Supplemental Figure 1C), including 4 of the 7 nuclear-encoded complex I core subunits (Figure 1D), were significantly downregulated in STZ diabetic $\mathrm{T}^{\mathrm{fl} / \mathrm{fl}}$ mice when compared with those in citrate-treated $\mathrm{T}^{\mathrm{fl} / \mathrm{fl}}$ controls. However, there were 0 differences between M-FoxO TKO and STZ-FoxO TKO. Statistical analysis of the fold-change ratio of STZ-diabetic $\mathrm{T}^{\mathrm{f} / \mathrm{l} / \mathrm{l}} /$ citrate-treated $\mathrm{T}^{\mathrm{f} / \mathrm{l}}$ versus the fold-change ratio of STZ-FoxO TKO/M-FoxO TKO showed that 3 of the 4 nuclear-encoded core subunits of complex I (Ndufs1, Ndufs3, and Ndufs8) and the mitochondrial-encoded mt-Nd6 were normalized after FoxO KO in muscle (Figure 1D). Among the other 78 OXPHOS subunits (excluding the 14 complex I core subunits), 23 were downregulated in STZ diabetic $\mathrm{T}^{\mathrm{f} / \mathrm{fl}}$ mice: 7 were from complex I (Supplemental Figure 1C), 3 from complex II (Supplemental Figure 1D), 1 from complex III (Supplemental Figure 1E), 4 from complex IV (Supplemental Figure $1 F)$, and 8 from complex V (Supplemental Figure 1G). Only 6 (Ndufb2, mt-Cytb, mt-Co1, mt-Atp6, Atp5f1, and Atp5l) of these 78 OXPHOS genes were significantly $(P<0.05)$ different when comparing fold-change ratio of STZ diabetic $\mathrm{T}^{\mathrm{f} / \mathrm{fl}} /$ citrate-treated $\mathrm{T}^{\mathrm{f} / \mathrm{fl}}$ with the fold-change ratio of STZ-FoxO TKO/M-FoxO TKO, indicating a predominant regulation of complex I core subunits by STZ diabetic $\mathrm{T}^{\mathrm{f} / \mathrm{fl}}$ mice and FoxOs. 
A

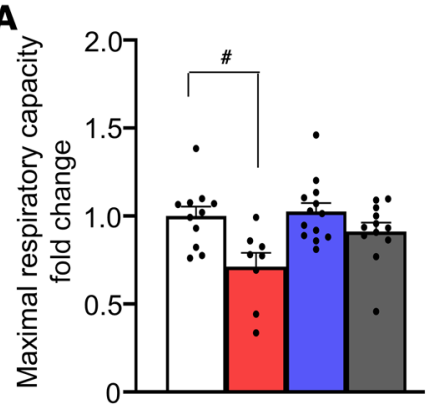

B

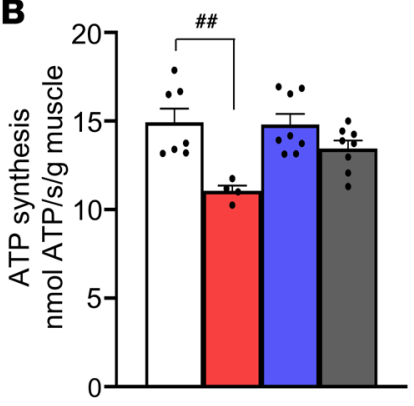

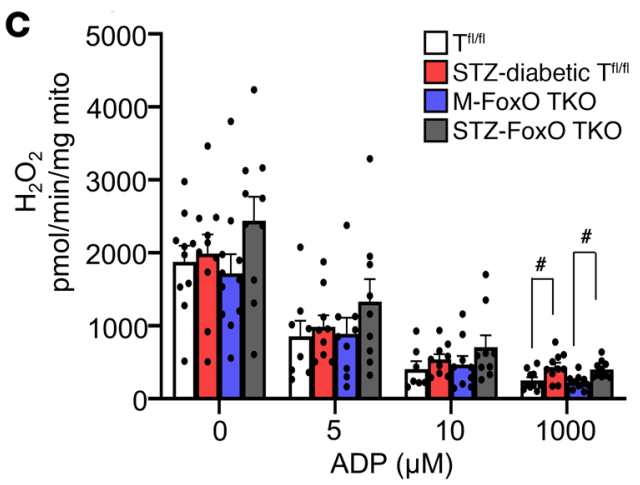

D

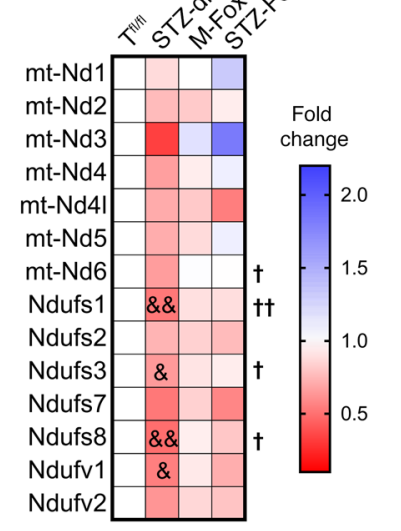

G

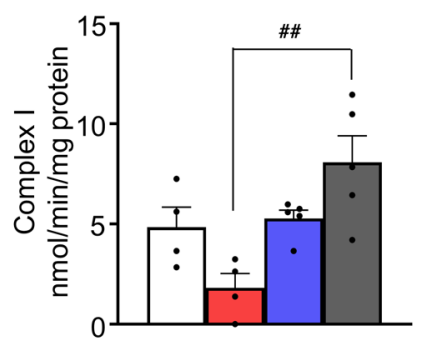

E

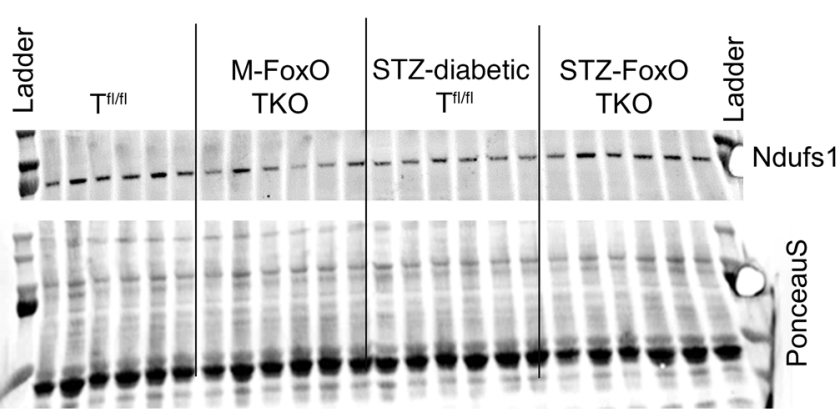

$\mathbf{F}$

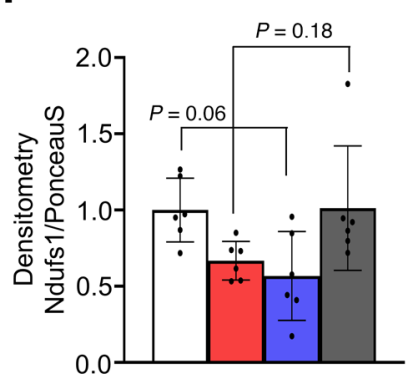

H

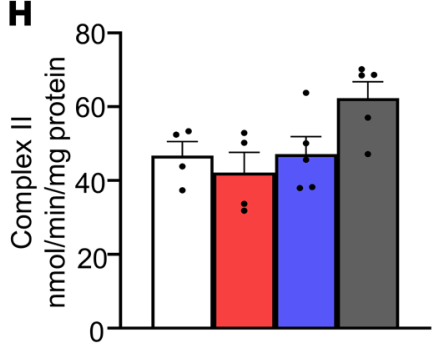

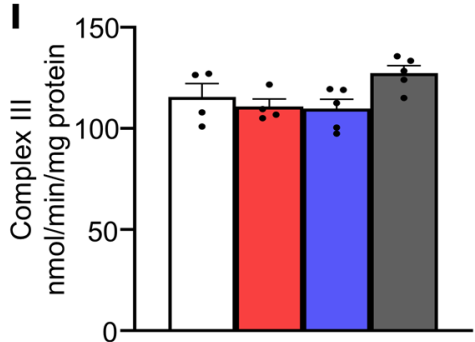

Figure 1. Deletion of FoxOs in muscle prevents complex I-mediated mitochondrial defects in response to insulin-deficient diabetes. (A and B) Maximal mitochondrial respiration (A) and ATP synthesis (B) using glutamate/malate/succinate substrates in mitochondrial-enriched fractions from quad/gast muscle from $\mathrm{T}^{\mathrm{fl} / \mathrm{fl}}$ controls, STZ diabetic $\mathrm{T}^{\mathrm{fl} / \mathrm{fl}}, \mathrm{M}$-FoxO TKO, and STZ FoxO-TKO mice $(n=4-12)$. (C) $\mathrm{H}_{2} \mathrm{O}_{2}$ production using glutamate/malate/succinate in isolated mitochondria from quad/gast muscle STZ-treated control and M-FoxO TKO mice at various concentrations of ADP $(n=8-10)$. (D) Heatmap of RNA-Seq fold changes of complex I core subunit transcripts in quad from STZ diabetic $\mathrm{T}^{\mathrm{fl} / \mathrm{fl}}$, M-FoxO TKO, and STZ-FoxO TKO compared with $\mathrm{T}^{\mathrm{fl} / \mathrm{fl}}$ controls $(n=4-6)$. \&FDR $<0.05$, \&\&FDR $<0.01 \mathrm{STZ}$ diabetic $\mathrm{T}^{\mathrm{fl} / \mathrm{fl}}$ vs. control; ${ }^{\dagger} P<0.05,{ }^{+\dagger} P<0.01$, ratio of STZ-FoxO TKO/M-FoxO TKO to STZ diabetic $\mathrm{T}^{\mathrm{fl} / \mathrm{fl} /}$ citrate-treated $\mathrm{T}^{\mathrm{fl} / \mathrm{fl}}$. (E and F) Western blot (E) and densitometry (F) of Ndufs1 (complex I protein) in STZ diabetic Tfl/fl and STZ-FoxO TKO quad with respective controls $(n=6)$. (G-I) OXPHOS complex I (G), complex II (H), and complex III (I) activity in gast from STZ diabetic Tfl/fl and STZ-FoxO TKO with respective controls. Results are represented as mean $\pm \mathrm{SEM}$. ${ }^{\#} P<0.05,{ }^{\# \#} P<0.01,2$-way ANOVA.

Western blots showed that levels of Ndufs1 protein tended to decrease in STZ diabetic $\mathrm{T}^{\mathrm{f} / \mathrm{ll}}$ and M-FoxO TKO, but Ndufs1 was higher in STZ-FoxO TKO muscle compared with STZ diabetic $\mathrm{T}^{\mathrm{f} / \mathrm{fl}}$, although this did not reach statistical significance (Figure 1, E and F). Other OXPHOS proteins were unaltered (Supplemental Figure $1, \mathrm{H}$ and I). Complex I activity tended to decrease in STZ diabetic $\mathrm{T}^{\mathrm{f} / \mathrm{fl}}$ mice and was significantly increased in STZ-FoxO TKO muscle compared with STZ diabetic $\mathrm{T}^{\mathrm{fl} / \mathrm{fl}}$ (Figure $1 \mathrm{G}$ ), with no changes in complex II or III activity (Figure 1, H and I). Thus, STZ diabetic $\mathrm{T}^{\mathrm{f} / \mathrm{fl}}$ mice had decreased muscle mitochondrial respiration, ATP synthesis, and complex I subunit expression and activity, and these defects were reversed following deletion of FoxOs in STZ FoxO TKO.
Muscle-specific deletion of IR alone or in combination with IGF1R decreases mitochondrial respiratory capacity and ATP production in permeabilized soleus fibers, but combined deletion of FoxO1, -3, and -4 with IR/IGF1R in M-QKO muscle does not alter mitochondrial function. We next examined the regulation of muscle mitochondrial bioenergetics by IR and IGF1R in the absence of systemic glucose or lipid abnormalities. We previously generated $\mathrm{M}-\mathrm{IR}^{-/}$,

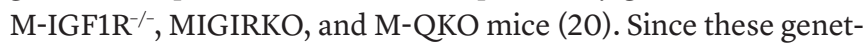
ically altered mice could not be generated from a single breeding scheme, we determined mitochondrial bioenergetics, biochemical assays, protein quantification, and mRNA measures separately in $\mathrm{M}-\mathrm{IR}^{-/-}$compared with $\mathrm{M}-\mathrm{IR}^{\mathrm{fl} / \mathrm{ll}}$ controls, $\mathrm{M}-\mathrm{IGF}_{\mathrm{R}} \mathrm{R}^{-/-}$compared 
with M-IGF1R ${ }^{\mathrm{fl} / \mathrm{fl}}$, MIGIRKO compared with $\mathrm{M}-\mathrm{IR} / \mathrm{IGF}^{\mathrm{fl} / \mathrm{fl}}$, and $\mathrm{M}-\mathrm{QKO}$ compared with $\mathrm{M}-\mathrm{Q}^{\mathrm{f} / \mathrm{fl}}$ controls (see Supplemental Methods for full details of our strategy for controlling for mixed genetic backgrounds). Real-time quantitative reverse-transcription PCR (qRT-PCR) of IR, IGF1R, and FoxO1, -3 , and -4 in these models demonstrated that the genes targeted for $\mathrm{KO}$ were decreased at the mRNA level in soleus muscle (Supplemental Figure 2, A-D). Western blot confirmed that IR was knocked out in $\mathrm{M}-\mathrm{IR}^{-/}$soleus muscle and that all 5 targeted genes in $\mathrm{M}-\mathrm{QKO}$ were decreased at the protein level relative to $\mathrm{M}-\mathrm{Q}^{\mathrm{f} / \mathrm{fl}}$ (Supplemental Figure 2E). In MIGIRKO mice, IR was decreased, but IGF1R protein was relatively preserved despite severe atrophy of the soleus (Figure 2B), which may reflect the fact that IGF1R is highly expressed in myoblasts (20) and possibly other nonmyocyte cells.

To confirm that deletion of IR and IGF1R in our models blocked downstream hormonal signaling, we injected supraphysiologic doses of insulin (5 units) or IGF-1 ( $1 \mathrm{mg} / \mathrm{kg}$ ) intravenously and measured Akt phosphorylation in soleus. Insulin-stimulated

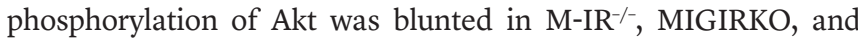
$\mathrm{M}-\mathrm{QKO}$ muscle when compared with control or $\mathrm{M}-\mathrm{IGF}_{\mathrm{R}} \mathrm{R}^{--}$muscle (Figure 2A). We have previously reported that MIGIRKO muscle has increased basal Akt levels, leading to increased basal Akt phosphorylation (19), but we observed no increase above basal with insulin stimulation. Similarly, IGF-1 injection in $\mathrm{M}-\mathrm{IGF}^{-1 /}$, MIGIRKO, and M-QKO mice showed reduced phosphorylation of Akt in soleus relative to that of IGF-1-treated control or $\mathrm{M}-\mathrm{IR}^{-1}$ mice. These changes in Akt phosphorylation were also reflected in phosphorylation of IR/IGF1R in soleus from our models (Supplemental Figure 2F), indicating that signaling downstream of IR and/ or IGF1R was appropriately blocked in our various mouse models.

Measurement of soleus mass showed that $\mathrm{M}^{-}-\mathrm{IR}^{-/}$was unchanged compared with controls, while $\mathrm{M}-\mathrm{IGF}^{-/-}$was increased (Figure 2B). However, soleus weight normalized to body weight was not different between $\mathrm{M}-\mathrm{IGF}^{-/-}$and $\mathrm{M}-\mathrm{IGF}^{\mathrm{I}} \mathrm{R}^{\mathrm{f} / \mathrm{Il}}$ (data not shown). In other muscle groups, $\mathrm{M}-\mathrm{IR}^{-/}$showed mild atrophy compared with controls, while $\mathrm{M}-\mathrm{IGF}_{\mathrm{R}} \mathrm{R}^{-/}$muscle size was unchanged (Supplemental Figure 2, G and H). MIGIRKO showed severe atrophy of soleus, while $\mathrm{M}-\mathrm{QKO}$ did not show changes in muscle mass relative to that of littermate controls (Figure 2B), a pattern that was also present in other muscle groups (Supplemental Figure 2, I and J).

To determine whether insulin and/or IGF-1 signaling directly affect muscle mitochondrial function via FoxOs, we measured respiration and ATP generation in saponin-permeabilized soleus fibers from $\mathrm{M}-\mathrm{IR}^{-/}$, M-IGF1R ${ }^{-/}$, MIGIRKO, and $\mathrm{M}-\mathrm{QKO}$ mice. Maximal soleus fiber respiration after addition of $1 \mathrm{mM}$ ADP was

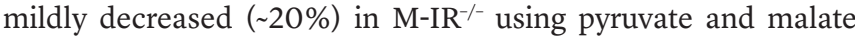
(PYR/M) as substrates (Figure $2 \mathrm{C}$ ). After sequential addition of the complex II substrate succinate and mitochondrial inhibitors rotenone (complex I), oligomycin (complex V), and the uncoupler carbonyl cyanide 4-(trifluoromethoxy)phenylhydrazone (FCCP), there were no significant differences in respiration between $\mathrm{M}-\mathrm{IR}^{-1}$ and $\mathrm{M}-\mathrm{IR}^{\mathrm{f} / \mathrm{fl}}$ controls. Mitochondrial respiration was unchanged between $\mathrm{M}-\mathrm{IGF}_{\mathrm{R}} \mathrm{R}^{-/}$and $\mathrm{M}-\mathrm{IGF} 1 \mathrm{R}^{\mathrm{fl} / \mathrm{ll}}$ in soleus using PYR/M (Figure 2D). Mitochondrial respiration was dramatically decreased in MIGIRKO soleus using PYR/M and remained decreased with sequential addition of succinate, rotenone, oligomycin, and
FCCP (Figure 2E). However, respiratory capacity was unchanged between $\mathrm{M}-\mathrm{QKO}$ and $\mathrm{M}-\mathrm{Q}^{\mathrm{f} / \mathrm{fl}}$ with pyruvate, succinate, and mitochondrial inhibitors (Figure $2 \mathrm{~F}$ ), indicating respiratory defects in MIGIRKO are not present with combined deletion of IR/IGF1R and FoxO1, -3, and - 4 in M-QKO when compared with their $\mathrm{M}-\mathrm{Q}^{\mathrm{f} / \mathrm{ll}}$ controls. The ATP synthesis rate with PYR/M trended lower in $\mathrm{M}-\mathrm{IR}^{-/}$without achieving statistical significance $(P=0.11)$ (Figure $2 \mathrm{G}$ ) and was unchanged in $\mathrm{M}-\mathrm{IGF}^{-/-}$(Figure $2 \mathrm{H}$ ). MIGIRKO fibers showed dramatic reduction compared with $\mathrm{M}-\mathrm{IR} / \mathrm{IGF}^{\mathrm{f}} \mathrm{R}^{\mathrm{f} / \mathrm{Il}}$ in ATP synthesis with PYR/M (Figure 2I), which was again unchanged in M-QKOs relative to controls (Figure 2J). Respiratory capacity with the fatty acid substrate palmitoyl-carnitine (PC)/ malate and ATP synthesis was not different in $\mathrm{M}^{-\mathrm{IR}^{-/}}$(Figure 2, $\mathrm{K}$ and $\mathrm{O}$ ) and $\mathrm{M}-\mathrm{IGF}_{\mathrm{R}} \mathrm{R}^{-/}$(Figure 2, L and $\mathrm{P}$ ) when compared with their respective floxed controls. Maximal respiration and ATP production were significantly decreased in MIGIRKO soleus fibers using PC/malate (Figure 2, M and Q), which was again unchanged in $\mathrm{M}-\mathrm{QKO}$ soleus fibers relative to controls (Figure 2, $\mathrm{N}$ and $\mathrm{R}$ ). Thus, long-term deficiency of IR alone mildly decreased, whereas combined deletion of both IR/IGF1R dramatically decreased, respiration and ATP production in soleus fibers with both PYR/M and $\mathrm{PC} /$ malate substrates. This diminished mitochondrial function in MIGIRKO was no longer present in $\mathrm{M}-\mathrm{QKO}$ muscle fibers, indicating FoxO transcription factors regulate mitochondrial function.

OXPHOS complex I and complex II activity are decreased in $M I G I R K O$ muscle and preserved in $M-Q K O$, but mitochondrial content remains unchanged. To determine whether the bioenergetic deficiencies when IR/IGF1R signaling is lost are due to decreases in mitochondrial content or defects in OXPHOS complex activity, we evaluated mitochondrial density by transmission electron microscopy (TEM) and mitochondrial enzyme expression and activity in muscle from MIGIRKO and M-QKO mice. MIGIRKO showed no differences compared with $\mathrm{M}-\mathrm{IR} / \mathrm{IGF}_{\mathrm{R}} \mathrm{R}^{\mathrm{f} / \mathrm{l} l}$ in mitochondrial area density quantitated in electron micrographs of the intermyofibrillar (IMF) (IMF mitochondria are located between sarcomere myofibrils) area of soleus (Figure 3, A and B). Since MIGIRKO mice have dramatic muscle atrophy, this means that the mitochondrial content is matched to the smaller IMF area. Loss of FoxOs in $\mathrm{M}-\mathrm{QKO}$ soleus did not change mitochondrial area density in comparison with that in $\mathrm{M}-\mathrm{Q}^{\mathrm{f} / \mathrm{fl}}$ controls (Figure 3, $\mathrm{C}$ and D). Citrate synthase (CS) activity in MIGIRKO soleus trended lower, but was not statistically changed (Figure 3E). However, both complex I and complex II activities measured in soleus from MIGIRKO were decreased (Figure 3, F and G), but CS, complex $\mathrm{I}$, and complex II activities were unchanged in $\mathrm{M}$-QKO (Figure 3, $\mathrm{H}-\mathrm{J})$. OXPHOS subunits Ndufs1 (complex I), Uqcrc2 (complex III), and Atp5a (complex V) were decreased in soleus from MIGIRKO and unchanged in $\mathrm{M}-\mathrm{QKO}$ mice (Figure 3, K-P).

Soleus is an oxidative muscle, but most skeletal muscle in mice is mixed glycolytic and oxidative, which show dramatic differences in OXPHOS expression and activity based on fibertype composition (31). To gain insight into IR/IGF1R and FoxO effects on mixed muscle, CS activity, OXPHOS complex activity, and expression were also evaluated in quad/gast muscle from MIGIRKO and M-QKO mice. In contrast to soleus, complex I activity was dramatically decreased, whereas complex II activity was mildly decreased in MIGIRKO quad/gast muscle (Supple- 

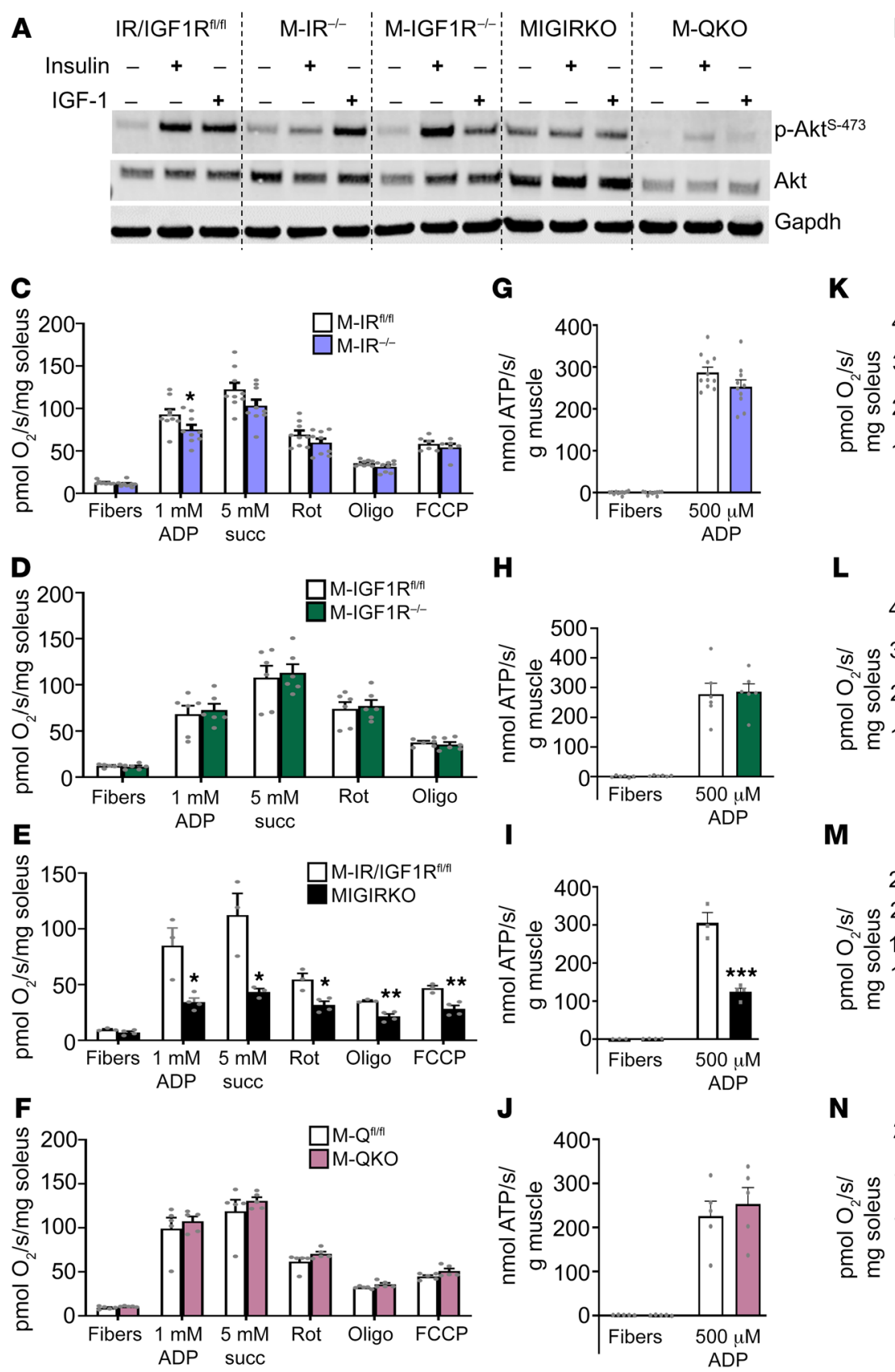

G

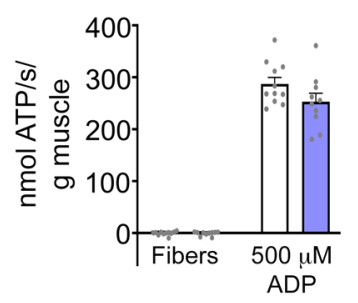

H
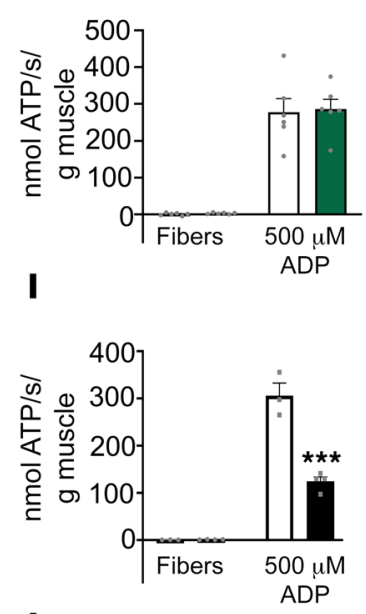

J

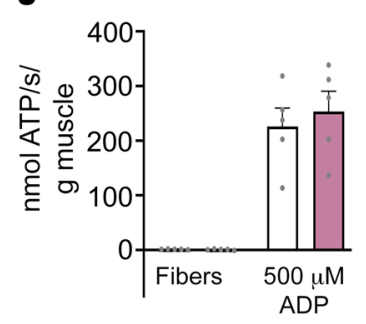

K

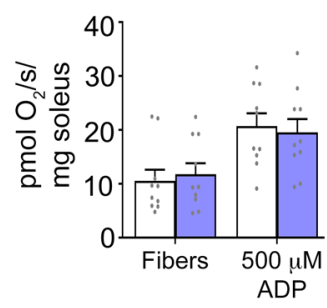

$\mathbf{L}$
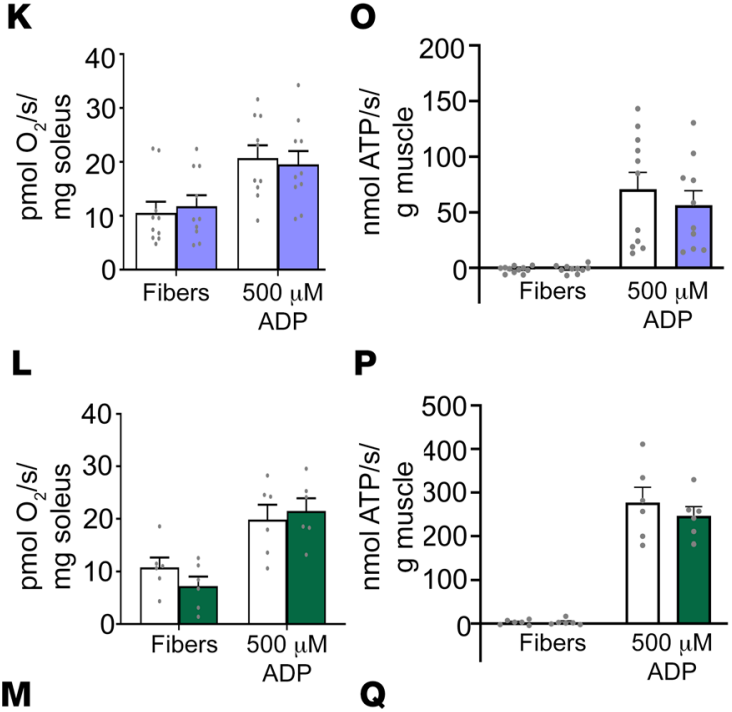

$\mathbf{P}$

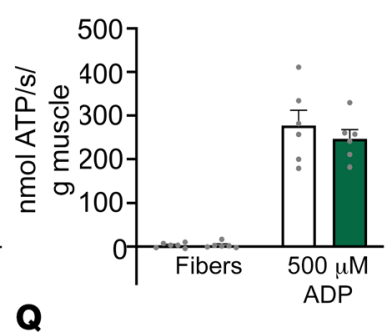

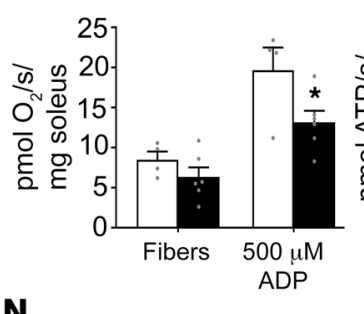

$\mathbf{N}$

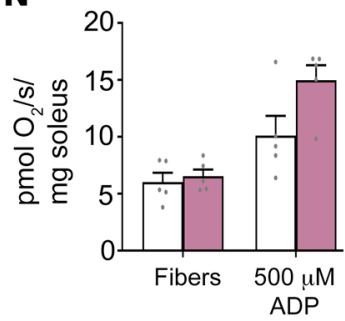

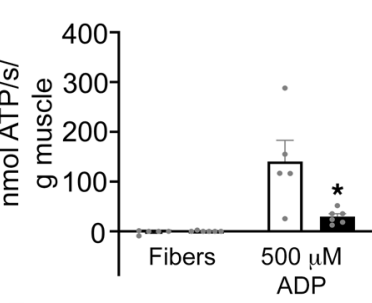

$\mathbf{R}$

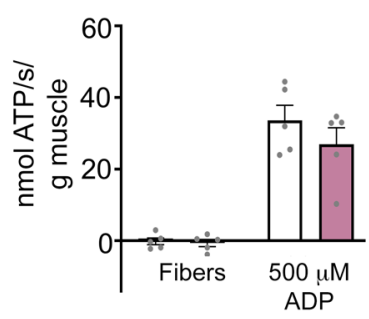

Figure 2. Muscle-specific deletion of IR alone or in combination with IGF1R decreases mitochondrial respiratory capacity and ATP production in permeabilized soleus fibers, but combined deletion of Fox01, -3, and -4 with IR/IGF1R in M-QKO muscle does not alter mitochondrial function. (A) Western

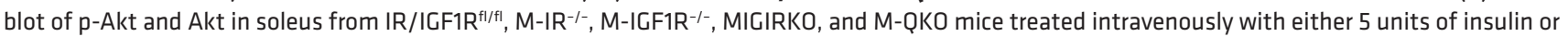
$1 \mathrm{mg} / \mathrm{kg}$ of IGF-1. (B) Muscle weight of soleus from M-IR ${ }^{-1-}, \mathrm{M}-\mathrm{IGF}_{\mathrm{R}}{ }^{-/-}$, MIGIRKO, and M-QKO $(n=3-6)$ relative to controls. (C-F) Respiratory capacity in soleus permeabilized muscle fibers with PYR/M substrates, and then with sequential addition of ADP, succinate (Succ), rotenone (Rot), oligomycin (Oligo),

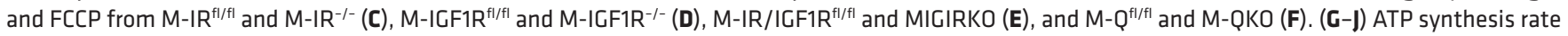

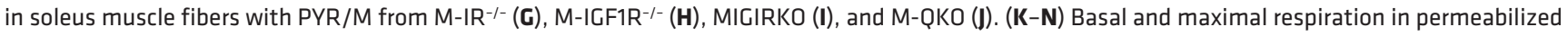

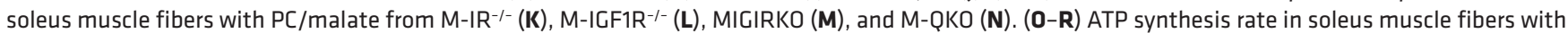

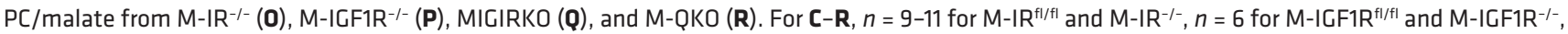
$n=3-6$ for M-IR/IGF1R ${ }^{f / / f 1}$ and MIGIRKO, and $n=5$ for M-Q $Q^{f / f 1}$ and M-QKO. Results are represented as mean \pm SEM. ${ }^{*} P<0.05 ;{ }^{* *} P<0.01 ;{ }^{* *} P<0.001$ vs. littermate control, $t$ test for 2 groups.

mental Figure 2, $\mathrm{K}$ and L). Complex III activity (Supplemental Figure 2M) and CS activity (data not shown) were unchanged in MIGIRKO quad/gast muscle. M-QKO mice again showed preserved mitochondrial enzyme activities relative to controls (Sup- plemental Figure 2, N-P). OXPHOS proteins were unchanged in quad/gast muscle from MIGIRKO and M-QKO when compared with their floxed controls (Supplemental Figure 2, Q-T). In summary, loss of both IR/IGF1R in oxidative soleus and mixed quad/ 

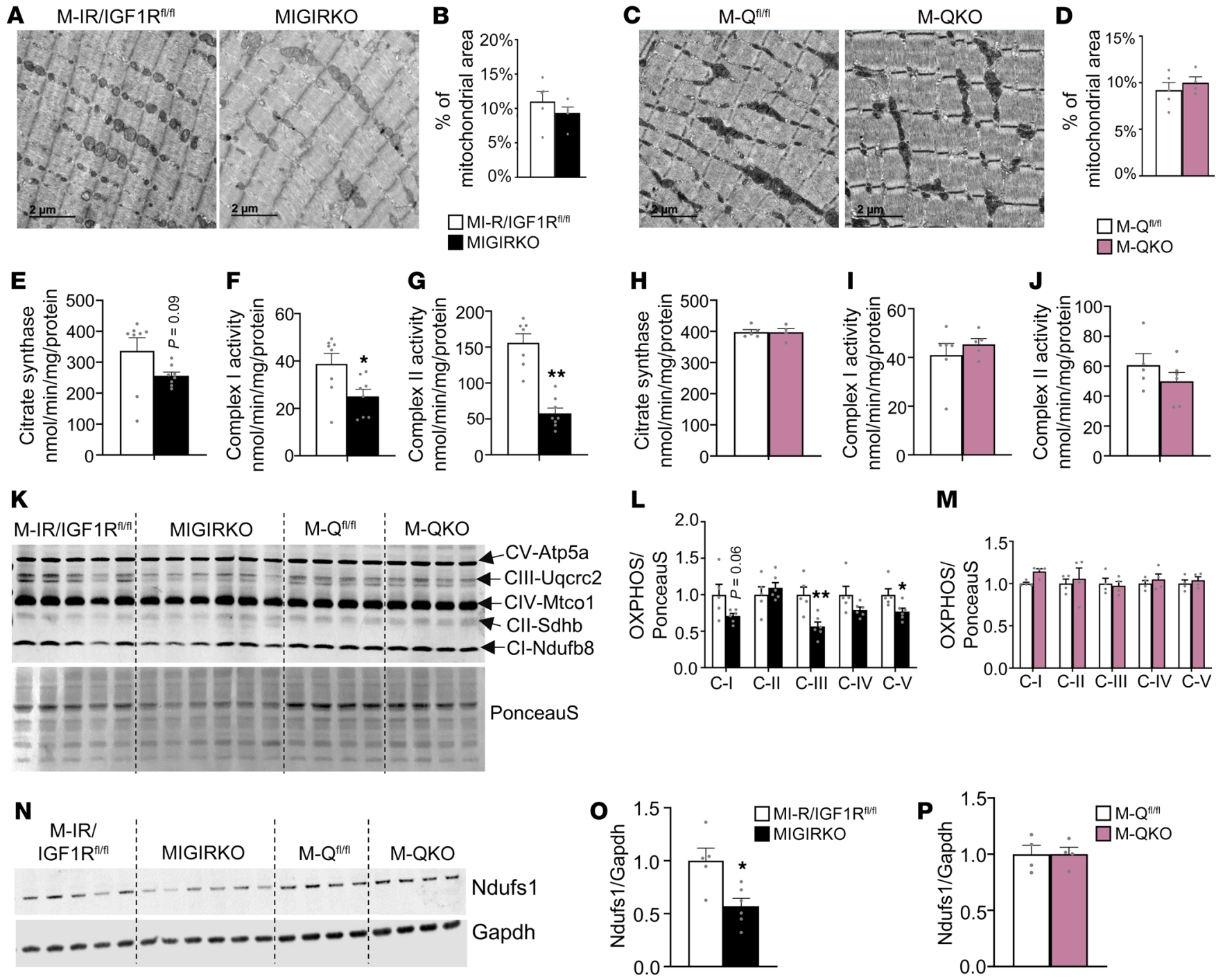

Figure 3. OXPHOS complex I and complex II activity are decreased in MIGIRKO soleus muscle and preserved in M-QKO, but mitochondrial content remains unchanged. (A and B) Electron micrographs (A) and quantified mitochondrial area density (B) in soleus from MIGIRKO and M-IR/IGF1R D) Electron micrographs (C) and quantitation of mitochondrial area density (D) in soleus from M-QKO and M-Qfl/fl $(n=4-6)$. (E-G) CS (E), OXPHOS complex I (F), and complex II (G) activity in soleus from MIGIRKO and M-IR/IGF1R ${ }^{\mathrm{fl} / \mathrm{fl}}(n=4-8)$. (H-J) CS (H), complex I (I), and complex II (J) activity in soleus from $M-Q K O$ and $M-Q^{f / f f l}(n=4-6)$. (K-M) Western blot (K) of OXPHOS proteins and densitometry in soleus from MIGIRKO (L) and M-QKO (M) with their respective controls $(n=3-6)$. (N-P) Western blot (N) of Ndufs1 and densitometry in soleus from MIGIRKO (0) and M-QKO (P) with respective controls $(n=4-6)$. Results are represented as mean $\pm \mathrm{SEM}$. ${ }^{*} P<0.05 ;{ }^{* *} P<0.01$, vs. littermate control, $t$ test for 2 groups. Scale bars: $2 \mu \mathrm{m}$.

gast muscle decreases both complex I and complex II activity in a FoxO-dependent manner, whereas mitochondrial mass, CS, and other OXPHOS complexes are less affected.

Loss of IR or both IR and IGF1R induces FoxO-dependent mitochondrial respiratory dysfunction in mixed muscle while increasing $\mathrm{H}_{2} \mathrm{O}_{2}$ production and protein carbonylation. To determine whether the mitochondrial dysfunction in oxidative soleus fibers from

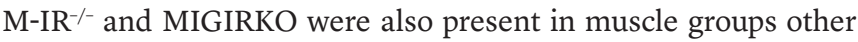
than soleus, we measured respiration and ATP production in mitochondria-enriched isolates from mixed quad/gast muscle of

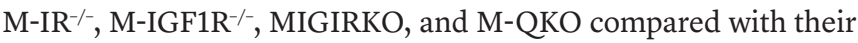
respective controls. Glutamate/malate-driven respiration with $1 \mathrm{mM}$ ADP was decreased by $34 \%$ in $\mathrm{M}^{-\mathrm{IR}^{-/}}$(Figure $4 \mathrm{~A}$ ) and by $49 \%$ in MIGIRKO (Figure 4C) mitochondria. This decrease was no longer observed in $\mathrm{M}-\mathrm{IR}^{-/}$or MIGIRKO mitochondria with subsequent addition of succinate, rotenone, and oligomycin, indicating a prominent complex I dysfunction in mixed muscle from both models that mimicked complex activity assays (Supplemental Figure 2, K-M). ATP synthesis rates in mitochondrial isolates were

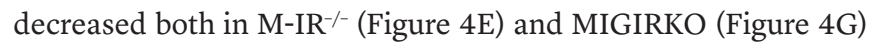
using glutamate/malate, but not after the addition of succinate. Mitochondrial respiratory capacity and ATP synthesis were not different between M-IGF1R $\mathrm{R}^{\mathrm{t} / \mathrm{l}}$ and M-IGF1R ${ }^{-/}$(Figure 4, B and F). Again, deletion of FoxOs in $\mathrm{M}-\mathrm{QKO}$ showed no changes in mitochondrial respiration (Figure 4D) and ATP production (Figure 4H) compared with $\mathrm{M}-\mathrm{Q}^{\mathrm{A} / \mathrm{fl}}$.

To determine whether mitochondrial dysfunction induced by loss of IR/IGF1R was linked to oxidative damage, we measured 
$\mathrm{H}_{2} \mathrm{O}_{2}$ emission, protein carbonylation, and expression of antioxidant mRNAs. $\mathrm{H}_{2} \mathrm{O}_{2}$ was increased by 2-fold in isolated mitochondria from $\mathrm{M}-\mathrm{IR}^{--}$with glutamate/malate/succinate substrate at low physiologic levels of $5 \mu \mathrm{M}$ ADP (Figure 4I), but was unchanged in the absence of ADP or with concentrations greater than $10 \mu \mathrm{M}$ ADP. In contrast, MIGIRKO mitochondria showed a dramatic 3to 5 -fold increase in $\mathrm{H}_{2} \mathrm{O}_{2}$ after addition of $5 \mu \mathrm{M}$ and $10 \mu \mathrm{M}$ ADP (Figure $4 \mathrm{~K}$ ), but no changes in $\mathrm{H}_{2} \mathrm{O}_{2}$ were seen in $\mathrm{M}-\mathrm{IGF}^{-/ /}$and $\mathrm{M}-\mathrm{QKO}$ mitochondria compared with their respective fl/fl controls (Figure $4, \mathrm{~J}$ and $\mathrm{L}$ ). With oligomycin $(0.01 \mathrm{mg} / \mathrm{ml}), \mathrm{H}_{2} \mathrm{O}_{2}$ production was increased in MIGIRKO mitochondria (Figure $4 \mathrm{~K}$ ). These increases in $\mathrm{H}_{2} \mathrm{O}_{2}$ production were associated with increased protein carbonylation in mitochondrial isolates from $\mathrm{M}-\mathrm{IR}^{-/-}$by $30 \%$ (Figure 4M), and carbonylation was also increased in MIGIRKO mitochondrial proteins (Figure $4 \mathrm{~N}$ ) and tissue homogenate (Supplemental Figure 3, A and B), whereas no changes were seen in $\mathrm{M}-\mathrm{QKO}$ compared with $\mathrm{M}-\mathrm{Q}^{\mathrm{f} / \mathrm{l}}$ (Figure $4 \mathrm{O}$ and Supplemental Figure 3, A and C). Increased ROS production is often accompanied by transcriptional changes to mitigate ROS toxicity. Glutathione peroxidase 3 (Gpx3) and Gpx4 mRNA levels were increased in quad from MIGIRKO. Additionally, Gpx4, superoxide dismutase 1 (Sod1), and thioredoxin 2 (Txnrd2) were increased in MIGIRKO soleus (Supplemental Figure 3D). These antioxidant genes were either significantly decreased (Gpx3) or unchanged in quad and soleus from $\mathrm{M}-\mathrm{QKO}$ (Supplemental Figure 3E).

Mitochondrial biogenesis and fission-fusion dynamics can regulate mitochondrial function. Mitochondrial biogenesis genes Ppara, Pprc1, Ppargc1a, Ppargc1b, and Tfam were not different between MIGIRKO and M-IR/IGF1R ${ }^{\mathrm{f} / \mathrm{l}}$ in either quad or soleus. Esrr- $\alpha$ was decreased in MIGIRKO quad, but not soleus, and Gabpb1 was decreased in MIGIRKO soleus, but not quad (Supplemental Figure 3F), demonstrating that the mRNA levels of these biogenesis mediators were not consistently regulated in MIGIRKO muscle. Mitochondrial biogenesis genes were not altered in M-QKO (Supplemental Figure 3G) except Ppargc1a, which was decreased in quad and tended to increase in soleus muscle. Pgc1- $\alpha$ is known as a master regulator of mitochondrial biogenesis, but we found no differences in protein levels between MIGIRKO, $\mathrm{M}-\mathrm{QKO}$, and their respective controls (Supplemental Figure 3, $\mathrm{H}-\mathrm{J})$. We also saw no changes in the mitochondrial fusion protein Opa1 (Supplemental Figure 3, K-M) and fission protein Drp1 (Supplemental Figure 3, N-P) in quad from MIGIRKO, M-QKO, and their respective $\mathrm{fl} / \mathrm{fl}$ controls. In summary, loss of IR/IGF1R decreased complex I-driven respiration in mitochondrial isolates from mixed quad/gast muscle, which is consistent with the results obtained in oxidative soleus fibers. This decrease in respiration was accompanied by increased oxidative stress, but could not be explained by changes in mitochondrial mass, mitochondrial biogenesis genes, or fission-fusion proteins.

Tamoxifen-inducible deletion of IR and IGF1R in muscle decreases muscle strength, soleus fiber respiration, and ATP production in concert with increased oxidative stress. $\mathrm{M}-\mathrm{IR}^{-/}, \mathrm{M}-\mathrm{IGF}_{\mathrm{R}} \mathrm{R}^{--}$, MIGIRKO, and $\mathrm{M}-\mathrm{QKO}$ mice are models of long-term deletion of IR, IGF1R, and/or FoxOs starting during embryonic development, which leads to a $60 \%$ decrease in muscle size in MIGIRKO. To determine whether mitochondrial impairment in MIGIRKO is secondary to developmental defects or represents a primary phe- nomenon, we performed muscle physiologic and mitochondrial bioenergetic assays on IND-IGIRKO mice, which were generated previously. IND-IGIRKO mice do not show recombination prior to tamoxifen treatment and displayed a near-total loss of IR and downregulation of IGF1R 3 weeks after tamoxifen treatment (20). In the current experiments, mRNA levels of IR were approximately $80 \%$ decreased, whereas IGF1R was approximately $50 \%$ decreased 3 weeks after tamoxifen both in soleus (Figure $5 \mathrm{~A}$ ) and quad (Supplemental Figure 4A) from IND-IGIRKO mice. IR and IGF1R protein levels were decreased in soleus (Figure 5, B and C), whereas quad muscle showed a significant decrease in IR, but IGF1R protein was mildly decreased (Supplemental Figure 4, $\mathrm{B}$ and C). Oxidative soleus (Figure 5D), mixed muscle, and glycolytic extensor digitorum longus (EDL) muscle weights (Supplemental Figure $4 \mathrm{D}$ ) were significantly decreased, by $20 \%$ to $30 \%$, in IND-IGIRKO mice 3 weeks after tamoxifen. This reduction in muscle mass was accompanied by decreased grip strength (Figure $5 \mathrm{E}$ ), treadmill test running time (Figure 5F), and speed at the time of exhaustion (Figure 5G) in IND-IGIRKO compared with tamoxifen-treated IR/IGF1R ${ }^{\mathrm{f} / \mathrm{f}}$ controls.

IND-IGIRKO showed a $24 \%$ to $28 \%$ decrease in maximal respiratory capacity in saponin-permeabilized soleus fibers with PYR/M and combined PYR/M and succinate (Figure $5 \mathrm{H}$ ). Maximal respiratory capacity after the addition of rotenone tended to decrease, but was no longer significant, indicating IND-IGIRKO muscle also showed a prominent complex I dysfunction. ATP synthesis rate was decreased 35\% (Figure 5I) with PYR/M in IND-IGIRKO soleus compared with controls. Soleus respiratory capacity also tended to decrease with PC/malate substrates (Supplemental Figure 4E), and ATP production was significantly decreased $(P<0.05)$ (Supplemental Figure $4 \mathrm{~F}$ ). Interestingly, respiratory capacity in glycolytic EDL fibers was not changed in IND-IGIRKO with PYR/M/succinate, but ATP synthesis was decreased at low $75 \mu \mathrm{M}$ ADP with PYR/M (data not shown). Respiratory capacity and ATP synthesis in mitochondrial isolates from quad/gast muscle of IND-IGIRKO were unchanged (Supplemental Figure 4, G and H). These data show that shortterm deletion of IR/IGF1R decreases muscle strength and exercise capacity and acutely impairs mitochondrial respiration and energy production in soleus muscle fibers.

Protein carbonylation and $\mathrm{H}_{2} \mathrm{O}_{2}$ production in quad was increased in IND-IGIRKO (Supplemental Figure 4, I-K). Furthermore, mRNA expression of antioxidant genes nuclear respiratory factor 1 (Nrf1), Sod1, and Txnrd1 and -2 were increased in INDIGIRKO soleus, and Cat, Gpx4, and Nrf1 were increased in INDIGIRKO quad (Supplemental Figure 4L) compared with controls. Thus, acute loss of IR/IGF1R in muscle decreased mitochondrial respiration and ATP production, particularly in oxidative soleus muscle, but also increased oxidative stress in mixed muscle groups, indicating that mitochondrial dysfunction occurs early and is not secondary to growth delay or other developmental abnormalities.

Acute loss of IR/IGF1R in muscle decreases complex I activity/ content and mitochondrial area density with minimal alterations in biogenesis genes. To determine whether acute loss of IR/IGF1R affects electron transport chain function or abundance, we measured OXPHOS complex proteins, complex activities, and biomarkers of mitochondrial content 3 weeks after tamoxifen treatment in IND-IGIRKO. Complex I activity (Figure 5J) in quad was 

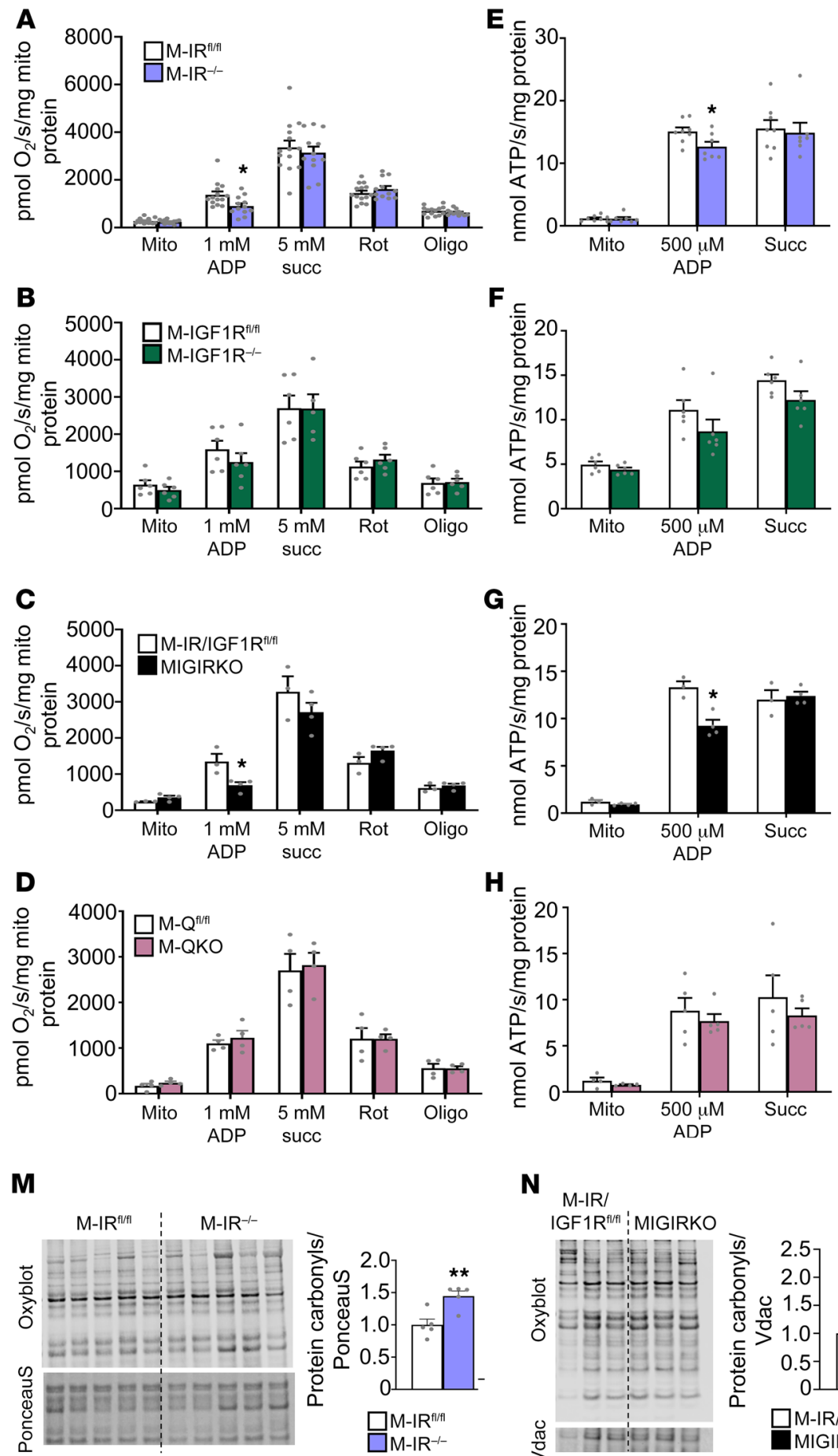
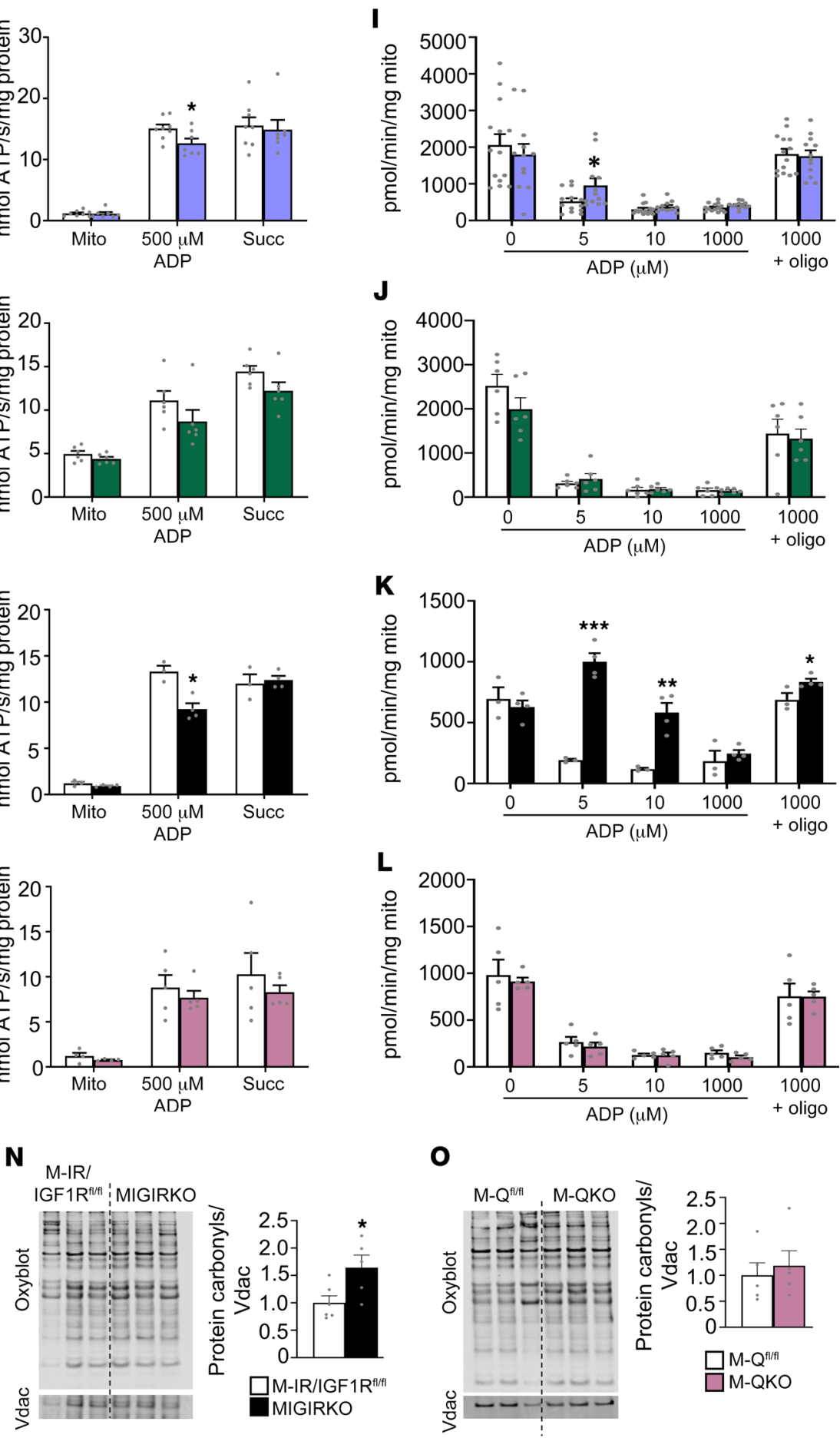

(

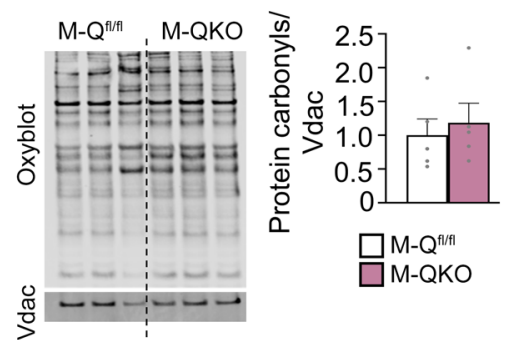

Figure 4. Loss of IR or both IR/IGF1R in mixed gast/quad muscle induces Fox0-dependent decline in mitochondrial respiratory function while increasing $\mathrm{H}_{2} \mathrm{O}_{2}$ production and protein carbonylation. (A-D) Basal and maximal respiration in quad/gast muscle mitochondria with glutamate/malate and

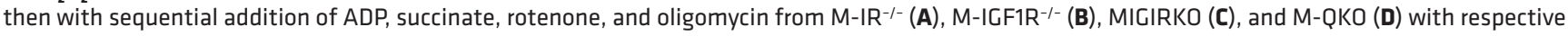
controls. (E-H) ATP synthesis rates in quad/gast muscle mitochondria from M-IR $\mathrm{R}^{-/-}(\mathbf{E}), \mathrm{M}-\mathrm{IGF1R}^{-/-}(\mathbf{F})$, MIGIRKO (G), and M-QKO (H) with respective con-

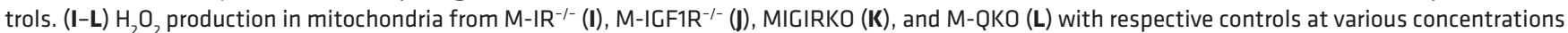
of ADP or with oligomycin. (M-0) Oxyblot images with quantification of protein carbonylation in mitochondria from M-IR ${ }^{-/-}(\mathbf{M})$, MICIRKO (N), and M-QKO (0) $(n=5-6)$ with controls. Mitochondria were isolated from mixed quad/gast muscle for each experiment. For $\mathbf{A}-\mathrm{L}, n=8-14$ for $M-I R^{f \mid / f l}$ and $M-I R^{-/-}, n=$ 6 for M-IGF1R ${ }^{\mathrm{fl} / \mathrm{fl}}$ and M-IGF1R ${ }^{-/-}, n=3-4$ for M-IR/IGF1R ${ }^{\mathrm{fl} / \mathrm{fl}}$ and MIGIRKO, and $n=4-5$ for $\mathrm{M}-\mathrm{Q}^{\mathrm{fl} / \mathrm{fl}}$ and $\mathrm{M}-\mathrm{QK} 0$. ${ }^{*} P<0.05 ;{ }^{*} P<0.01$; ${ }^{* * *} P<0.001$ vs. littermate control, $t$ test for 2 groups. 


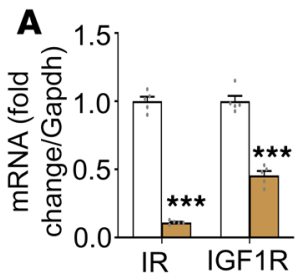

B

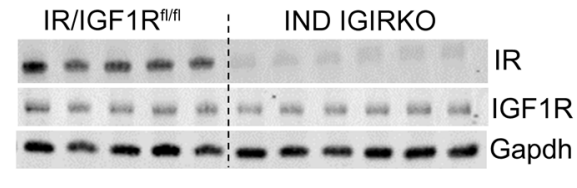

C

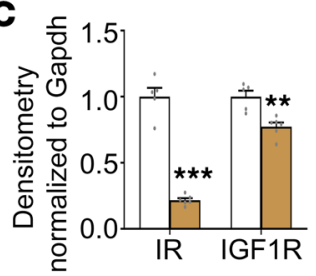

D

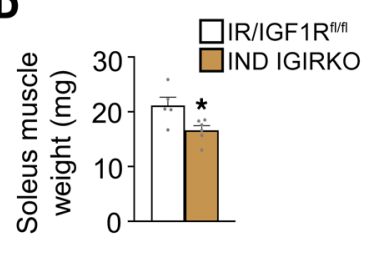

E

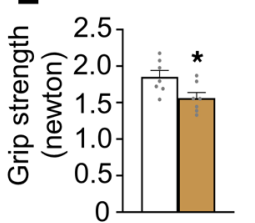

J

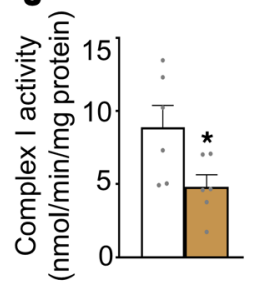

$\mathbf{N}$

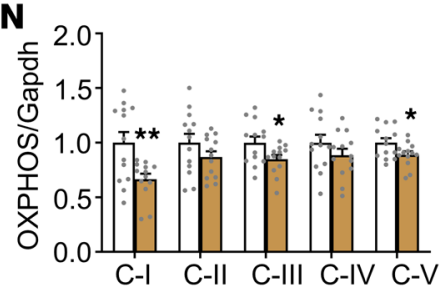

$\mathbf{Q}$

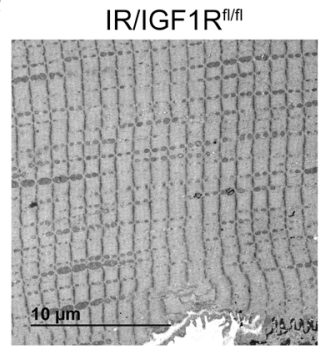

$\mathbf{F}$

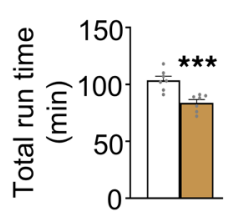

K

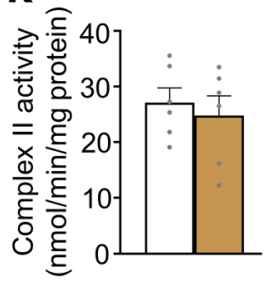

0

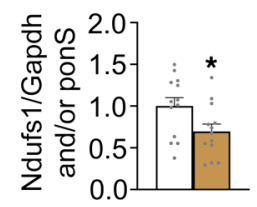

G

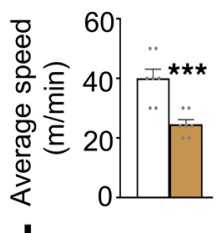

$\mathbf{L}$

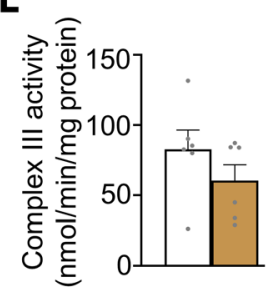

$\mathbf{P}$
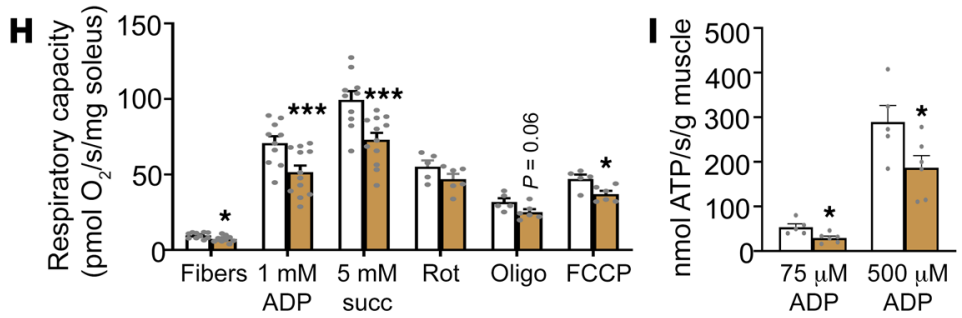

M

M IR/IGF1R ${ }^{\mathrm{fl/fI}}:$ IND IGIRKO
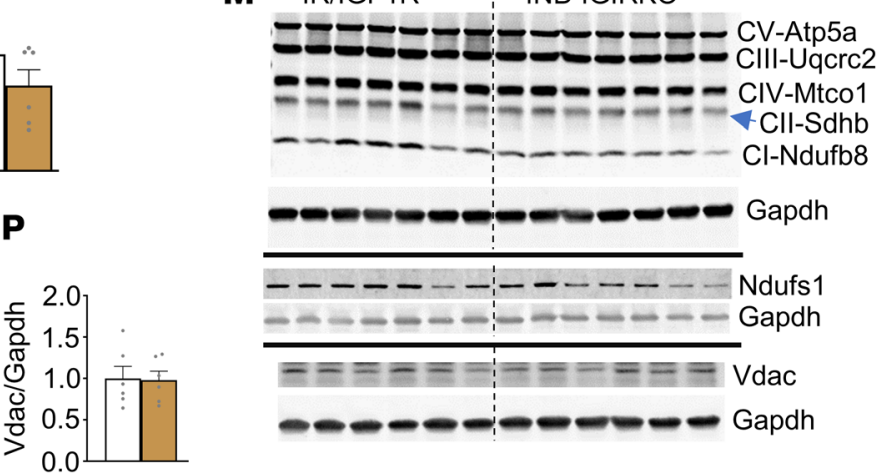

- - - - $-\cdots-\ldots$ Ndufs 1 - $------1-\cdots-\cdots-$ Gapdh

$=-=-=-1=-=-=-\mathrm{Vdac}$

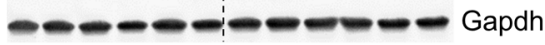

Figure 5. Tamoxifen-inducible deletion of IR and IGF1R in muscle decreases muscle strength, soleus fiber respiration, ATP production, complex I activity, OXPHOS proteins, and mitochondrial area density. (A) mRNA levels of IR and ICF1R in soleus muscle 3 weeks after a 5-day course of tamoxifen treat-

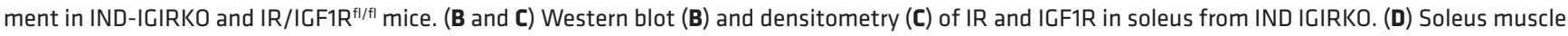
weight in IND-IGIRKO and IR/IGF1R fI/fI mice $(n=5-6)$. (E and F) Forelimb grip strength $(\mathbf{E})$, treadmill run time $(\mathbf{F})$, and speed at the time of exhaustion $(\mathbf{C})$ in IND-IGIRKO and IR/IGF1R fI/fI $(n=7)$. (H) Respiratory capacity in permeabilized soleus fibers with PYR/M, then subsequent addition of ADP, succinate, rotenone, oligomycin, and FCCP in IND-IGIRKO and IR/IGF1R ${ }^{\mathrm{fl} / \mathrm{fI}}(n=10-12)$. (I) ATP generation in soleus fibers with PYR/M from IND-IGIRKO ( $n=5$-6). (J-L) OXPHOS complex I in quad (J) and complex II (K) and complex III (L) activity in gast from IND-IGIRKO and IR/IGF1R ${ }^{\mathrm{fl} / \mathrm{fI}}(\boldsymbol{n}=6)$. (M-P) Western blot $(\mathbf{M})$ with densitometry of OXPHOS proteins (N), Ndufs1 (0), and Vdac (P) in quad from IND-IGIRKO and IR/IGF1R fl/fI ( $n=13$ for OXPHOS and Ndufs1; $n=6$ for Vdac). ( $\mathbf{Q}$ and $\mathbf{R}$ ) Electron micrographs (I) and quantitation of mitochondrial area density (J) in soleus from IND-IGIRKO and IR/IGF1R ${ }^{\mathrm{fl} / \mathrm{fI}}(n=5-6)$. Results are represented as mean \pm SEM. ${ }^{*} P<0.05 ;{ }^{* *} P<0.01 ;{ }^{* *} P<0.001$ vs. littermate control, $t$ test for 2 groups. ponS, PonceauS for normalization.

decreased by $50 \%$ in IND-IGIRKO. In contrast, complex II and complex III (Figure 5, K and L) activities in gast from IND-IGIRKO were not different. Activity of CS was not decreased in quad muscle homogenate (data not shown). Unlike in MIGIRKO, expression of complex I subunits Ndufb8 and Ndufs1 was robustly decreased in quad from IND-IGIRKO compared with IR/IGF1R ${ }^{\mathrm{f} / \mathrm{fl}}$, while other OXPHOS complex subunits were less affected (Figure 5,
$\mathrm{M}-\mathrm{O}$ ). Voltage-gated anion channel (Vdac) protein was not altered (Figure 5, M and P), suggesting that overall mitochondrial protein content was not different in quad from IND-IGIRKO. To further evaluate mitochondrial content, we determined mitochondrial area of IMF mitochondria by TEM in soleus muscle (Figure 5Q). This analysis showed an approximately 35\% decrease (Figure $5 \mathrm{R})$ in IMF mitochondrial volume after acute loss of IR/IGF1R 
in IND-IGIRKO. Despite the decrease in mitochondrial area in soleus, we did not observe a consistent change in the expression of mitochondrial biogenesis genes. Levels of Ppargc1b mRNA were decreased in IND-IGIRKO soleus compared with controls, while Pprc1 was increased (Supplemental Figure 4M). In quad, Gabpb1 and Pprc1 were increased in IND-IGIRKO, while Tfam was decreased (Supplemental Figure 4M). Ppargc1a tended to decrease both in soleus and in quad but these changes did not reach statistical significance. These data indicate that acute loss of IR/IGF1R in muscle is associated with decreases in mitochondrial content and complex I activity, but does not correlate with a coordinate regulation of mitochondrial biogenesis genes.

Muscle mitochondrial protein synthesis and mitochondrial fusion proteins are decreased in IND-IGIRKO. The insulin/IGF-1/FoxO signaling axis is a critical regulator of muscle proteostasis and protein synthesis (12). We first measured total muscle protein synthesis rates using the SUnSET method (32). The decline in mitochondrial content by acute loss of IR/IGF1R in IND-IGIRKO was accompanied by decreased protein synthesis in muscle tissue homogenate as well as mitochondrial isolates (Supplemental Figure $4, \mathrm{~N}-\mathrm{P}$ ). Muscle protein synthesis is regulated by the mTORC1 pathway (33), but we observed a paradoxical activation in mTOR signaling in IND-IGIRKO, demonstrated by an increase in phosphorylation of 4ebp1 (Supplemental Figure 4, Q and R) with no change in S6 phosphorylation (Supplemental Figure 4, Q and S). Alternatively, fission/fusion dynamics may influence mitochondrial content and IND-IGIRKO showed a significant decrease in Opa1 (Supplemental Figure 4, T and U), but no change in Drp1 protein (Supplemental Figure 4, T and V).

Mitophagy flux is not increased by loss of $I R / I G F 1 R$ in vivo and in vitro. Both perinatal and acute deletion of IR/IGF1R in muscle increases autophagy flux (the flow of proteins/cargo through the degradative process in lysosomes) via FoxOs (20), so we reasoned that mitophagy (the flow of mitochondrial proteins/cargo through autophagy) would be activated in IR/IGF1R deletion models and could contribute to the mitochondrial changes observed. Bnip3, a marker of mitophagy, was increased in MIGIRKO quad and tended to decrease in M-QKO (Figure 6, A and B). Bnip3 was also increased in mitochondrial-enriched fractions of IND-IGIRKO (Figure 6, C and D), which could indicate Bnip3-dependent mitophagy is activated in these muscles.

To determine whether mitophagy flux is increased, MIGIRKO, IND-IGIRKO, and control mice were treated with saline or colchicine, a known autophagy inhibitor in muscle (34). First, we confirmed that bulk autophagy flux measured by accumulation of LC3-II after colchicine was appropriately increased in INDIGIRKO (Supplemental Figure 5, A-C). Bnip3 protein was increased in $\mathrm{IR} / \mathrm{IGF}^{\mathrm{f}} \mathrm{f}^{\mathrm{l} / \mathrm{fl}}$ after treatment with colchicine compared with saline (Supplemental Figure 5, D and E), indicating basal mitophagy flux could be measured in control mice. In contrast, there were no increases in Bnip3 expression between saline and colchicine treatment in IND-IGIRKO and MIGIRKO mice (Figure 6, E and F, and Supplemental Figure 5, D and E). We assessed and found minimal differences in another mitophagy marker, Pink1, in IR/IGF1R ${ }^{\mathrm{f} / \mathrm{fl}}$ mice treated with or without colchicine (Supplemental Figure 5, F and G). However, Pink1 is a poor marker of muscle mitophagy in vivo $(35,36)$. These results indicate mitophagy flux was not increased and was actually impaired after deletion of IR/ IGF1R, which is contrary to our hypothesis.

To develop a cell model for insulin regulation of mitochondrial function and mitophagy, we used C2C12 mouse skeletal muscle-derived cell lines, which have been extensively used to study metabolic diseases (37). C2C12 myotubes were pretreated with or without insulin and carbonyl cyanide m-chlorophenylhydrazone (CCCP) (a known mitophagy inducer) for 4 hours on day 7 of differentiation prior to metabolic analysis using the Seahorse XF24 analyzer. These results showed that insulin increases oxygen consumption rate (OCR) in C2C12 myotubes and that 4-hour pretreatment with CCCP (washed out immediately prior to mitochondrial analysis) decreased basal and maximal respiration, but insulin cotreatment with CCCP partially rescued the decline in basal and maximal respiration (Supplemental Figure 6A).

Given the challenges with quantifying mitophagy markers, we took advantage of a mitophagy biosensor, mito-Keima $(38,39)$. IR/IGF1R ${ }^{\mathrm{f} / \mathrm{l}}$ primary myotubes were infected with adenoviral-Cre (Ad-Cre) or control adenoviral-luciferase (Ad-Luc) and with adeno-mito-Keima to monitor mitophagy. By day 6 of differentiation, IR and IGF1R were completely knocked out (Figure 6G and and Supplemental Figure 6B). Western blots for LC3-II showed that autophagy was induced after CCCP treatment in both Ad-Luc and Ad-Cre cells, but insulin was able to mitigate the LC3-II accumulation only in Ad-Luc cells (Supplemental Figure 6, C and D). CCCP increased the mitophagy index (ratio of mito-Keima fluorescence at $550 \mathrm{~nm}$ vs. $450 \mathrm{~nm}$ ) equally in Ad-Luc and Ad-Cre cells (Figure $6 \mathrm{H}$ ), indicating deletion of IR/IGF1R did not enhance or prevent the capacity to increase mitophagy in response to CCCP. Insulin treatment of Ad-Luc and Ad-Cre cells did not change the mitophagy index either when cells were treated alone or with CCCP (Figure $6 \mathrm{H}$ ), indicating insulin does not regulate mitophagy in these cells. Thus, loss of IR/IGF1R in primary myotubes did not change basal or CCCP-induced mitophagy in vitro.

We utilized MitoTimer to monitor mitophagy in vivo, as it can serve as a proxy of mitochondrial degradation and hence, mitophagy (40, 41). We coelectroporated MitoTimer with the lysosomal protein marker LAMP1-YFP and mitochondrially targeted blue fluorescent protein (mitoBFP) into tibialis anterior (TA) muscle of IND-IGIRKO and control mice. Colocalization of MitoTimer with mitoBFP (Supplemental Figure 6E) indicated we achieved appropriate expression of the MitoTimer in mitochondria. Although we previously showed mild increases in $\mathrm{H}_{2} \mathrm{O}_{2}$ and carbonylation, acute loss of IR/IGF1R in INDIGIRKO did not show differences in the average red/green ratio compared with that in controls (Figure 6, I and J). Distribution of red/green ratio of MitoTimer images in TA from IND-IGIRKO and IR/IGF1R ${ }^{\mathrm{f} / \mathrm{fl}}$ confirmed there were no differences in MitoTimer oxidation (Supplemental Figure 6F). In agreement with the expression of Bnip3 after colchicine, colocalization of red MitoTimer puncta (red/green ratio >2.0) and LAMP1-YFP was unchanged in IND-IGIRKO (Figure 6, I and K), indicating mitophagy was not activated in these mice. Thus, the mitochondrial dysfunction observed when IR and IGF1R are deleted in muscle is not due to increased mitophagy and clearance of mitochondria, but instead may be related to a reduction in mitophagy flux or alternative mechanisms. 
A
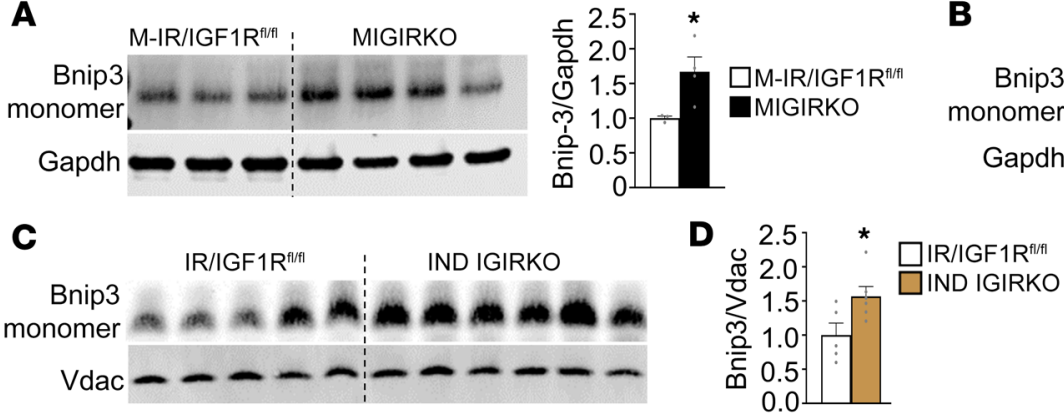

\section{E}

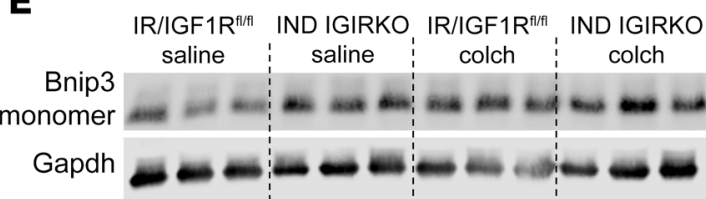

B

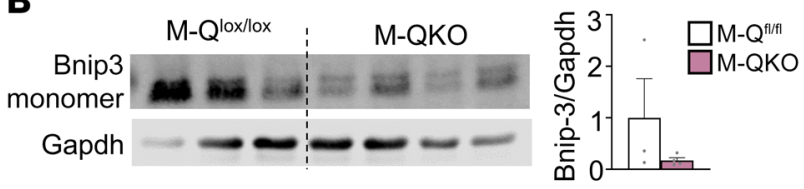

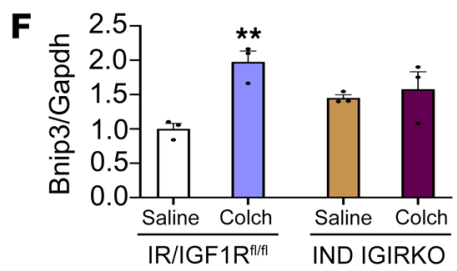

I
H

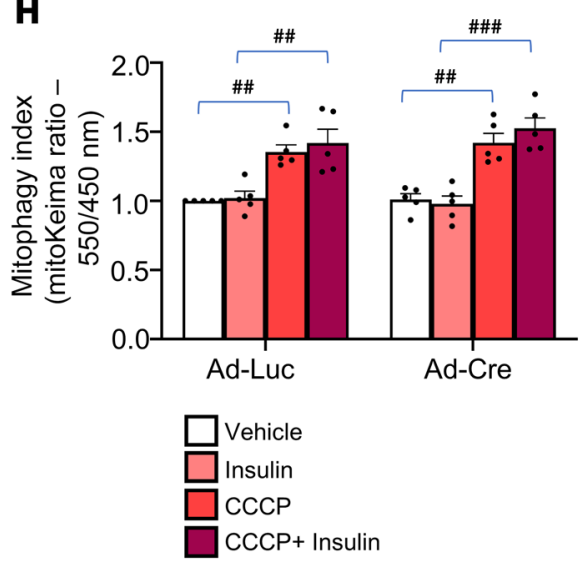

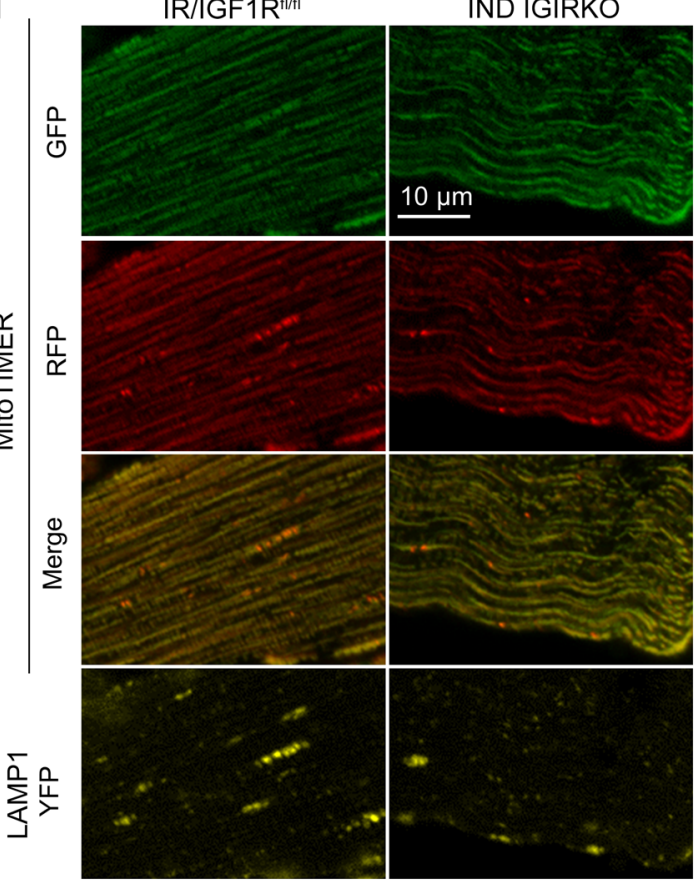

G Ad-Luc: Ad-Cre

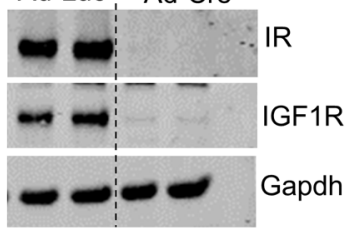

J

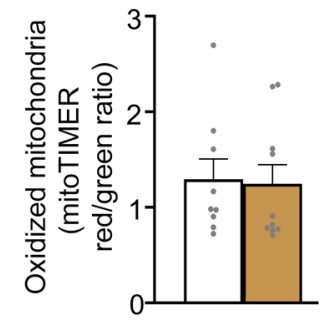

$\mathbf{K}$

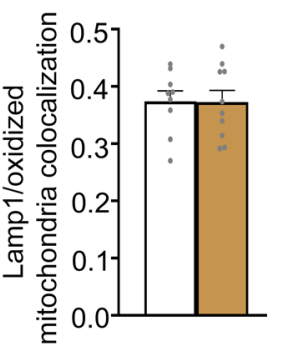

$\square$ IR/IGF1R IIND IGIRKO

Figure 6. Mitophagy flux is not increased by loss of IR/IGF1R in vivo and in vitro. (A and B) Western blot images and densitometry of Bnip3 in quad tissue homogenate from MIGIRKO (A) and M-QKO (B) with respective controls ( $n=3-4)$. (C and $\mathbf{D})$ Western blot images of Bnip3 (C) and densitometry (D) in mitochondrial isolates in quad/gast muscle from IND-IGIRKO with respective controls $(n=5-6)$. (E and F) Western blot image from IND-MIGIRKO (E) with densitometry (F) of Bnip3 in quad tissue homogenate after 2-day colchicine or saline treatment $(n=3-4)$. (C) Western blot of IR and IGF1R from IR/ IGF1R $^{f l / f l}$ primary myotubes on day 6 of differentiation after Ad-Cre or Ad-Luc. (H) Measurement of mitophagy in primary myotubes at day 7 of differentiation, previously infected with Adeno-mito-Keima, Ad-Cre, and Ad-Luc. Myotubes were treated with or without insulin and CCCP for 4 hours prior to imaging for mitophagy index ( $n=3$ experiments). Mitophagy index is displayed as the ratio of mito-Keima fluorescence using $550 \mathrm{~nm}$ excitation versus $450 \mathrm{~nm}$ excitation (550/450 nm). (I) Confocal microscopy analysis of TA muscle from day-21 IND-IGIRKO and control mice after coelectroporation with the mitochondrial oxidation probe, MitoTimer, and lysosomal LAMP1-YFP. Merged panels are also presented in Supplemental Figure 6E. Original magnification, $\times 40$. (J and $\mathbf{K})$ Quantification of mitochondrial oxidation measured as MitoTimer red/green ratio (I) and colocalization of oxidized mitochondria (MitoTimer red/green ratio $>2$ ) and LAMP1-YFP measured as Manders' coefficient $(\mathrm{J})(n=9-10)$. Results are represented as mean \pm SEM. ${ }^{*} P<0.05 ;{ }^{* *} P<$ 0.01 vs. littermate control, $t$ test for 2 groups. ${ }^{\# \#} P<0.01 ;{ }^{\# \#} P<0.001$, 3-way ANOVA.

Deletion of IR/IGF1R in muscle decreases complex I core subunits in a FoxO-dependent manner and disrupts complex I-dependent supercomplex assembly and activity. We demonstrated thus far that loss of insulin and IGF-1 action in muscle leads to a predominant defect in complex I-driven respiration. To determine whether the decline in mitochondrial function by loss of IR/IGF1R is transcriptionally regulated, we performed RNA-Seq analysis in quad from MIGIRKO and M-QKO mice. We performed a more detailed analysis of OXPHOS subunits and found a broad downregulation of OXPHOS with 28 genes out of 92 significantly (FDR $<0.01$ ) 
decreased in MIGIRKO (Figure 7, A and B). None of these were significantly changed in $\mathrm{M}-\mathrm{QKO}$ versus $\mathrm{M}-\mathrm{Q}^{\mathrm{fl} / \mathrm{ll}}$ controls, but a mitochondrial encoded gene, $\mathrm{mt}-\mathrm{Nd} 3$, was decreased. Although $\mathrm{M}-\mathrm{QKO}$ and MIGIRKO are generated from separate mouse lines (see Supplemental Methods), principal component analysis (PCA) from RNA-Seq results showed that IR/IGF1R ${ }^{\mathrm{f} / \mathrm{fl}}$ and $\mathrm{M}-\mathrm{Q}^{\mathrm{fl} / \mathrm{ll}}$ controls clustered together, but were distinct from MIGIRKO and M-QKO (Supplemental Figure 7A). Twenty-four of the 28 OXPHOS genes that were downregulated in MIGIRKO were significantly increased when comparing M-QKO to MIGIRKO by an FDR of less than 0.01 (Supplemental Table 3). When analyzing subunits by individual complexes, we observed that 10 of the 14 complex I core subunits (71\%) were highly repressed in MIGIRKO, but not in M-QKO (Figure 7A). Three genes (Sdha, Sdhc, and Sdhd) of complex II, 2 genes (mt-Cytb and Uqcrc2) of complex III, 3 genes (mt-Co1, Cox5a, and Cox 5b) of complex IV, and 5 genes (Atp5a1, Atp5b, Atp5c1, Atp5h, and ATP5s) of complex $\mathrm{V}$ were decreased in MIGIRKO (Figure 7B). Acute deletion of IR/ IGF1R in IND-IGIRKO also showed decreases in mRNA levels of subunits of complexes I-V both in soleus (Supplemental Figure 7B) and quad (Supplemental Figure 7C). In summary, both perinatal and acute loss of IR/IGF1R decreased OXPHOS subunit expression, particularly the core subunits of complex I, but deletion of FoxOs in conjunction with IR/IGF1R in muscle did not lead to this transcriptional decline.

Blue native polyacrylamide gel electrophoresis (BN-PAGE) in IND-IGIRKO showed that only complex I content was significantly decreased in quad (Figure 7, C and D). Supercomplexes III+IV, supercomplexes I+IV, supercomplexes I+III, and supercomplexes I+III+IV were all significantly decreased in quad from INDIGIRKO compared with controls (Figure 7, C and E). Soleus muscle showed a more robust decrease in complexes I, II, III, and IV and supercomplexes I+IV (Supplemental Figure 7, D-F) in INDIGIRKO. In contrast, MIGIRKO quad only showed a decrease in complex II and no decreases in content of complex I and supercomplexes (Supplemental Figure 7, G-I). Interestingly, in-gel activity of complex I in MIGIRKO was not different from that of IR/IGF1R controls, but the background staining in the lane, possibly due to increased oxidative damage, may have altered this measurement (Supplemental Figure 7, J-L). M-QKO did not show differences in levels of complexes or supercomplexes (Supplemental Figure 7, M-O). Finally, in-gel activity of quad muscle from IND-IGIRKO showed free complex I was unchanged, whereas complex I-containing supercomplex activity was decreased in supercomplexes I+IV, supercomplexes I+III, and supercomplexes I+III+IV in IND-IGIRKO compared with controls (Figure 7, F and $\mathrm{G})$. Taken together, these data indicate that IR/IGF1R signaling in muscle regulates mitochondrial complex I subunit transcription as well as complex/supercomplex function by suppressing FoxO transcription factors.

\section{Discussion}

Decreased muscle strength is a critical indicator of compromised muscle health and contributes to physical disability in diabetes $(1,4)$. Decreased energy production and mitochondrial abnormalities have been reported in muscle from diabetic patients $(5,6,9)$, but whether insulin action, which is decreased in both type 1 and type 2 diabetes (42), plays a direct role in regulation of mitochondrial respiration or merely alters substrate delivery/utilization is not fully understood. This study investigated the contribution of FoxOs to muscle mitochondrial metabolism in insulin-deficient diabetes and the relative contribution of IR, IGF1R, and FoxOs in the regulation of mitochondrial metabolism using muscle-specific KO mouse models. We showed that loss of insulin in STZ diabetic $\mathrm{T}^{\mathrm{fl} / \mathrm{fl}}$ mice decreases muscle mitochondrial respiration, ATP production, and complex I activity and that these changes were reversed with FoxO deletion in STZ-FoxO TKO. Furthermore, we demonstrated that loss of IR is sufficient to impair mitochondrial metabolism, whereas combined loss of IR/ IGF1R leads to a dramatic mitochondrial decline. These bioenergetic impairments were again reversed with deletion of FoxO1, -3 , and -4 . Detailed analysis revealed that IR/IGF1R signaling specifically regulates complex I activity, subunit expression, and supercomplex content in a FoxO-dependent manner. Thus, loss of insulin action in diabetes or by deletion of IR or both IR/IGF1R in muscle decreases complex I-driven mitochondrial respiration and supercomplex assembly, at least in part by FoxO-mediated repression of mitochondrial genes.

A growing body of evidence indicates that type 1 and type 2 diabetes are associated with muscle mitochondrial respiratory defects, but the cause and effect relationship remains controversial. Early studies identified mitochondrial defects in muscle from insulin-resistant humans $(5,6,43,44)$ at times in the disease when muscle from type 2 patients showed defects in signaling downstream of insulin (42). Several studies in mice supported the idea that obesogenic diets can cause muscle mitochondrial dysfunction $(45,46)$, leading to the idea that mitochondrial dysfunction can induce insulin resistance. However, other studies in mice and humans indicated that diet-induced obesity is not sufficient to cause mitochondrial decline $(47,48)$ and that nutrient overload (49) or loss of insulin action, as in type 1 or type 2 diabetes, was the key driver of mitochondrial abnormalities in muscle (15).

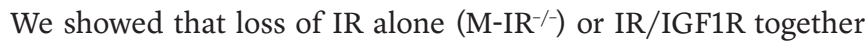
(MIGIRKO) in muscle is sufficient to cause defects in maximal respiratory capacity and ATP production and induces oxidative damage in the absence of nutrient overload or systemic glycemic abnormalities. We further identified that FoxO transcription factors mediate mitochondrial defects when insulin action is lost. These findings were confirmed in insulin-deficient STZ-diabetic models and may help explain the mitochondrial defects seen in patients with type 1 diabetes (9). The action of insulin suppresses FoxOs via the PI3K/Akt pathway, but other studies showed that muscle-specific deletion of the $\mathrm{p} 110 \alpha$ subunit of PI3K caused muscle atrophy, which is associated with increased mitochondrial mass, respiration, and ROS generation (50), while deletion of the p85/p55 subunits of PI3K led to muscle atrophy and features of metabolic syndrome accompanied by minimally decreased CS activity (51). Whether separate pools of PI3K interact with different upstream activators or may act in distinct subcellular compartments of the myocyte to control mitochondrial biology needs further investigation. Nonetheless, our results indicate that impaired insulin action in muscle is sufficient to cause mitochondrial dysfunction and that these metabolic defects are not present with deletion of FoxOs in M-QKO. 
A

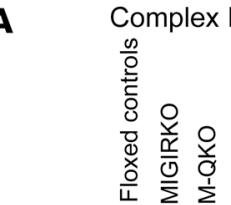

B

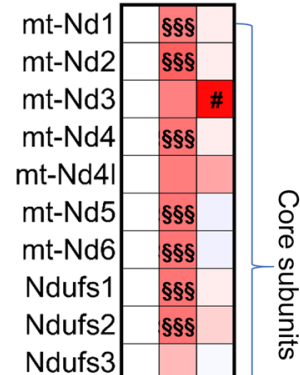

Ndufs7

Ndufs8 $8 \$ \S$

Ndufv1 $\S \S$

Ndufv2 $\$ \S$

Ndufa1

Ndufa10

Ndufa11

Ndufa12

Ndufa13

Ndufa2

Ndufa3

Ndufa4

Ndufa4l2

Ndufa5 $\$ \S$

Ndufa6

Ndufa7

Ndufa8

Ndufa9

Ndufab1

Ndufb10

Ndufb11

Ndufb2

Ndufb3

Ndufb4

Ndufb5

Ndufb6

Ndufb7

Ndufb8

Ndufbs

Ndufc 1

Ndufc2

Ndufs4

Ndufs5

Ndufs6

Ndufv3
옹

음

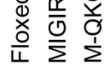
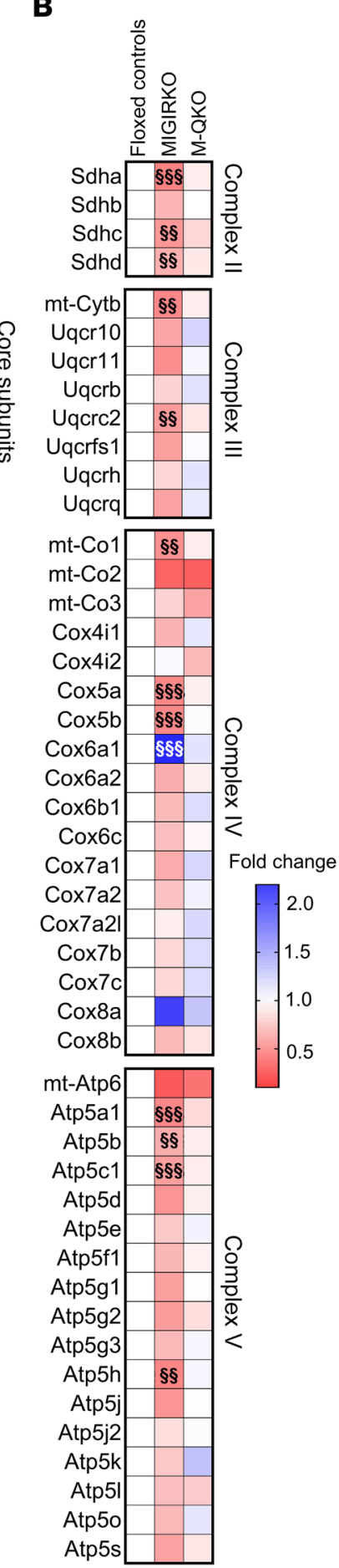

C

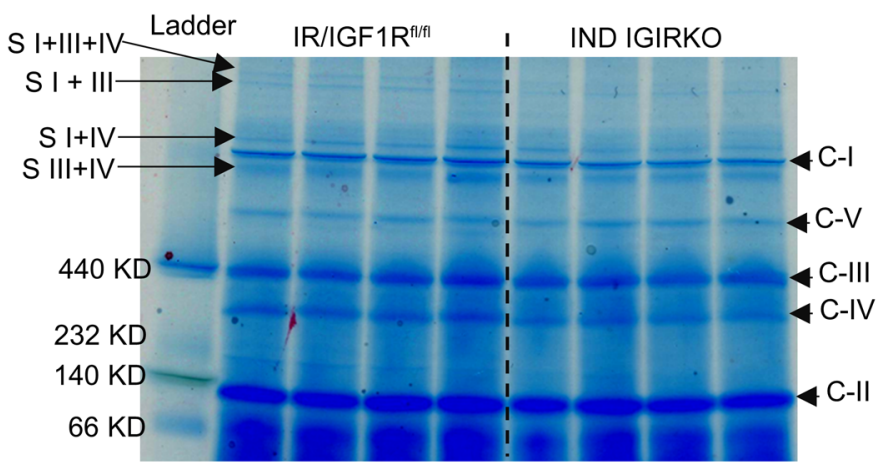

D

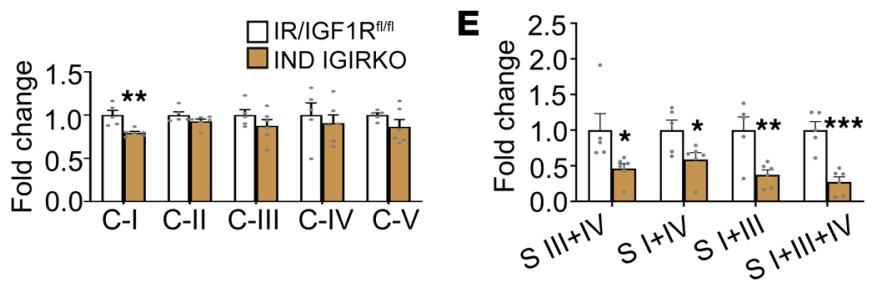

F

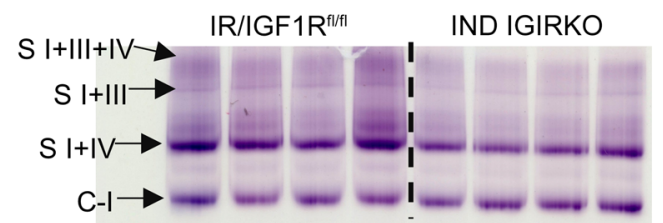

G

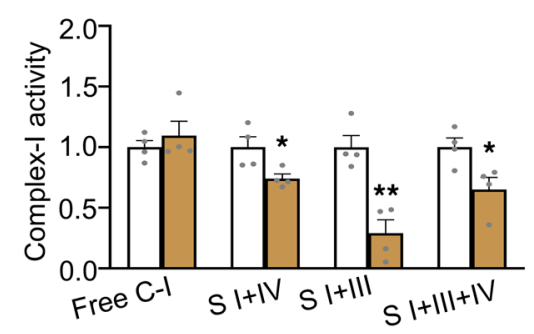

Figure 7. Deletion of IR/IGF1R in muscle decreases complex I core subunits in a FoxO-dependent manner and disrupts complex I-dependent supercomplex assembly and activity. (A and B) Heatmaps displaying RNA-Seq transcript levels of OXPHOS subunits in mixture of quad and gast from MIGIRKO and M-QKO compared with floxed controls. Heatmap of complex I, including core complex subunits (A). Heatmap of complexes II-V (B) $(n=4-6)$. Floxed

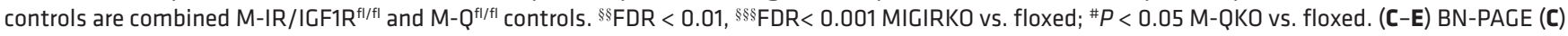
with densitometry of individual complexes I-V (D) and supercomplexes (E) in quad from IND-IGIRKO and IR/IGF1R fl/fl $(n=5-6)$. C, complex. (F and G) Clear native gel showing complex I in-gel activity (F) and densitometry (G) in quad from IND-IGIRKO and IR/IGF1R ${ }^{\mathrm{fl} / \mathrm{fl}}(n=4)$. Results are represented as mean \pm SEM. ${ }^{*} P<0.05 ;{ }^{* *} P<0.01 ;{ }^{* *} P<0.001$ vs. littermate control, $t$ test for 2 groups. 
An important question we addressed is whether IR/IGF1R deletion induced mitophagy, since the IR/IGF1R/FoxO signaling axis is a critical regulator of autophagy $(12,20,28,29)$. Increased mitophagy can improve mitochondrial function and fission-fusion dynamics in various disease conditions (52). We showed that deletion of IR/IGF1R in muscle did not change mitophagy (biosensor experiments) or could even impair mitophagy flux as measured by decreased Bnip3 accumulation despite robust upregulation of autophagy flux measured by LC3 accumulation. Thus, the mitochondrial dysfunction observed when IR and IGF1R are deleted in muscle is not due to increased mitophagy, but may be influenced by a reduction in mitophagy flux by mechanisms that are distinct from regulation of bulk autophagy.

A critical finding from this study is that IR and IGF1R regulate complex I-mediated respiration and that this regulation occurred in both oxidative soleus and mixed muscle groups that exhibit very different OXPHOS composition (31). Complex I (NADH oxidase) is the largest complex of OXPHOS, containing 44 different nuclear and mitochondrial-encoded subunits (53), and is the foundation for supercomplexes containing I/IV, I/III, or I/III/IV, which increase efficiency of electron transfer. Our results indicate that the subunits of the core complex of complex I are specifically strongly repressed after acute loss of IR/IGF1R and are restored when FoxOs are deleted. FoxOs have previously been shown to regulate the OXPHOS subunit Ndufv2 across species (54), which fits with the fact that mitochondrial dysfunction in STZ-diabetic mice is restored with insulin treatment (15). Our results also aligned with a recent study in which the authors observed that deficiency of the complex I subunit ND-49 (mammalian NDUFS2) induces ROS generation via activation of the mTOR pathway (55), but the direct connection between complex I defects and mTOR activation was not fully explored in our own study. Furthermore, the mitochondrial respiratory defects were coincident with ROS production and

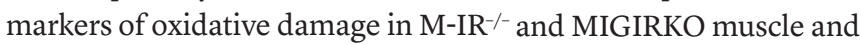
were not present in $\mathrm{M}-\mathrm{QKO}$. FoxOs are critical regulators of oxidative stress pathways (56), and this adds another layer of complexity to the mitochondrial regulation that will require further investigation. Thus, we identify what we believe is a new regulatory capacity of insulin/IGF-1 signaling in muscle. Insulin suppresses FoxO activation to maintain mitochondrial complex I subunit transcription as well as complex/supercomplex function, which correlates with improved strength and prevention of muscle atrophy.

Our study has several limitations. First, the use of a mixed background strain, rather than backcrossing onto a pure genetic background, can cause significant variability in metabolic studies (57). Given that the QKO line has 11 alleles (Acta-Cre and 10 targeted floxed alleles in 5 genes), backcrossing is practically challenging. Nonetheless, similar mitochondrial findings in MIGIRKO and IND-IGIRKO generated on different mixed backgrounds do lend credence to our findings, but do not change the contribution of genetic heterogeneity as a source of variance. The use of isolated mitochondria may cause heterogeneity, as subsarcolemmal and IMF mitochondria can have specialized function (58), although we aimed to control this with our isolation techniques (see Supplemental Methods) and use of permeabilized fibers. Additionally, physical activity is associated with muscle mitochondrial function and we did not assess this in each mouse prior to mitochondrial studies. We previously published that spontaneous activity of MIGIRKO was decreased 30\% in the dark cycle (19) and MIGIRKO mice were unable to perform spontaneous wheel running (20); thus, mitochondrial defects in MIGIRKO may be partly due to inactivity. Indeed, we cannot disassociate changes in mitochondrial function from changes in muscle size, and future work with time-course studies will be needed to decipher mechanisms that regulate these phenomena.

In conclusion, our results identify an important mechanism through which insulin and IGF-1 inactivate FoxOs to regulate the expression of complex I core subunits, mitochondrial respiration, and supercomplex assembly in muscle. Future identification of mechanisms by which FoxOs regulate complex I may hold promise for therapeutic interventions to prevent muscle weakness induced by diabetes and its related complications.

\section{Methods}

Animal care and use. Male mice were used in all animal studies

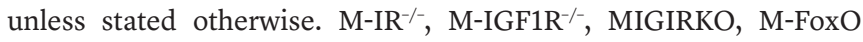
$\mathrm{TKO}, \mathrm{M}-\mathrm{QKO}$, and IND-IGIRKO mice were generated as previous-

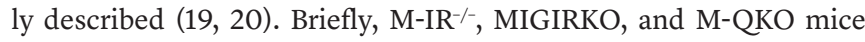
were generated using Acta1-Cre (stock 006149; Jackson Laboratory). IND-IGIRKO mice were generated using muscle-specific tamoxifen-inducible HSA-Cre-ER ${ }^{\mathrm{T} 2}$ (59). Each mouse line was maintained in a separate colony, and floxed littermates were used as controls in each experiment. For full details of mouse generation and design to control for mixed background strain, see Supplemental Methods.

Free access to food and water was available to all animals. For inducible KO of IR/IGF1R, $100 \mathrm{mg} / \mathrm{kg}$ tamoxifen (T5648; SigmaAldrich) dissolved in peanut oil was intraperitonially injected 5 times over 6 days in 10-week-old mice for the inducible recombination of IR and IGF1R in IND-IGIRKO. STZ was used to create a model of insulin-deficient diabetes. $\mathrm{T}^{\mathrm{f} / \mathrm{l} l \mathrm{l}}$ and $\mathrm{M}$-FoxO TKO mice were fasted overnight and then injected with a single high dose $(150 \mathrm{mg} / \mathrm{kg})$ of STZ dissolved in $100 \mathrm{mmol} / \mathrm{L}$ citrate buffer ( $\mathrm{pH} 4.5$ ) or citrate buffer alone as a control. Food was immediately provided after injections. Mice were monitored for hyperglycemia 3, 7,12, and 15 days after treatment, and only those mice that displayed blood glucose of more than 300 $\mathrm{mg} / \mathrm{dL}$ after 9 to 12 days after STZ were used for experiments.

Mitochondrial functional assays. Mitochondrial respiratory capacity and ATP generation were measured in saponin-permeabilized soleus fibers, EDL fibers, and mitochondrial-enriched fractions from quad/gast muscle (See Supplemental Methods for isolation technique) using a high-resolution Oroboros Oxygraph, as described previously (60-62). Briefly, harvested soleus and/or EDL muscle was immediately placed in an ice-cold buffer $\mathrm{X}$ containing $7.23 \mathrm{mM} \mathrm{K}_{2}$ EGTA, 2.77 mM CaK${ }_{2}$ EGTA, $20 \mathrm{mM}$ imidazole, $0.5 \mathrm{mM}$ DTT, $20 \mathrm{mM}$ taurine, 5.7 mM ATP, $14.3 \mathrm{mM}$ creatine monophosphate, $6.56 \mathrm{mM} \mathrm{MgCl}_{2}-6 \mathrm{H}_{2} \mathrm{O}$, and $50 \mathrm{mM}$ 2-[N-morpholino] ethane sulfonic acid (MES), with a $\mathrm{pH}$ of 7.1. The fibers were manually dissociated, separated into 3 bundles using needles under the dissecting microscope, and permeabilized in buffer X supplemented with $50 \mu \mathrm{g} / \mathrm{ml}$ saponin for 40 minutes on ice. Permeabilized fibers were incubated in buffer $\mathrm{Z}$ containing $105 \mathrm{mM}$ K-MES, $30 \mathrm{mM} \mathrm{KCl}, 10 \mathrm{mM} \mathrm{KH}_{2} \mathrm{PO}_{4}, 5 \mathrm{mM} \mathrm{MgCl}_{2}-6 \mathrm{H}_{2} \mathrm{O}, 2.5 \mathrm{mg} / \mathrm{ml}$ BSA, 1 mM EGTA, $20 \mathrm{mM}$ Blebbistatin, and $20 \mathrm{mM}$ creatine monohydrate, $\mathrm{pH}$ 7.4, on ice until further use. For respiration measurements, fibers were incubated in $2.5 \mathrm{ml}$ of buffer $\mathrm{Z}$ at $30^{\circ} \mathrm{C}$, and respiratory 
capacity was determined using Oroboros Oxygraph with the various combination of substrates, including malate $(2 \mathrm{mM})$, pyruvate $(5 \mathrm{mM})$, PC $(20 \mu \mathrm{M})$, glutamate $(5 \mathrm{mM}), \mathrm{ADP}$ (as indicated), succinate $(5 \mathrm{mM})$, rotenone $(10 \mu \mathrm{M})$, oligomycin $(10 \mu \mathrm{g} / \mathrm{ml})$, and FCCP $(10 \mu \mathrm{M})$. ATP production was measured by converting ATP into NADPH by addition of glucose ( $4 \mathrm{mM})$, NADP $(200 \mu \mathrm{M}), 50 \mu \mathrm{M}$ P1, P5-Di (adenosine-50) pentaphosphate pentasodium salt (MilliporeSigma D4022), and 10 $\mu \mathrm{L} / \mathrm{mL}$ of a hexokinase/glucose-6-phosphate dehydrogenase mixture (MilliporeSigma, 10127825001) to modified buffer Z (105 mM K-MES, $30 \mathrm{mM} \mathrm{KCl}, 10 \mathrm{mM} \mathrm{KH}_{2} \mathrm{PO}_{4}, 5 \mathrm{mM} \mathrm{MgCl}-6 \mathrm{H}_{2} \mathrm{O}, 0.5 \mathrm{mg} / \mathrm{ml} \mathrm{BSA}$, $1 \mathrm{mM}$ EGTA, pH 7.4). ATP production was monitored in a Horiba fluorometer $\mathrm{Ex} / \mathrm{Em}(345 \mathrm{~nm} / 460 \mathrm{~nm}) . \mathrm{H}_{2} \mathrm{O}_{2}$ production in mitochondrial isolates from quad/gast muscle was determined using $75 \mu \mathrm{g} / \mathrm{ml}$ of mitochondrial protein in a respiration buffer containing $120 \mathrm{mM} \mathrm{KCl}$, $5 \mathrm{mM} \mathrm{KH} \mathrm{PO}_{4}, 2 \mathrm{mM} \mathrm{MgCl}, 1 \mathrm{mM}$ EGTA, 3 mM HEPES, 0.3\% BSA (fatty acid free), $\mathrm{pH}$ 7.2, as described previously (62). Respiration was determined in mitochondrial isolates using $50-75 \mu \mathrm{g} / \mathrm{ml}$ of mitochondrial protein in either respiration buffer or in modified buffer Z. See Supplemental Methods for full details.

In vivo and in vitro mitophagy assessment. In vitro mitophagy quantification was performed using mito-Keima adenovirus (see Supplemental Methods for details) in primary muscle cells isolated from IR/ IGF1R floxed muscle. Recombination of IR and IGF1R was done using Ad-Cre or Ad-Luc.

In vivo mitophagy was assessed using MitoTimer as previously described $(40,41)$. MitoTimer is a mitochondria-targeted green fluorescent protein that shifts to red fluorescent when oxidized. MitoTimer (Addgene, catalog 52659), LAMP1-YFP (Addgene, catalog 1816), and mitoBFP (Addgene, catalog 49151) constructs were electroporated into TA muscle by somatic gene transfer. Briefly, electroporation was performed 14 days after tamoxifen treatment in IND-IGIRKO. Muscle was extracted and fixed 1 week after electroporation, which was 21 days after tamoxifen treatment in these mice, and longitudinal sections were prepared and imaged on a confocal microscope (see Supplemental Methods).

Acquired images were deconvolved, background-subtracted, and thresholded using Image s software (NIH) with default algorithms to segment mitochondria. The sum of $488 \mathrm{~nm}$ and $543 \mathrm{~nm}$ images was used as a mask to encompass the total mitochondrial signal. MitoTimer ratiometric analysis was carried out by dividing red by green images. Ratiometric data are expressed as the pixelwise relative frequency of the ratio (histogram, Supplemental Figure 6F). For MitoTimer and LAMP-YFP colocalization analysis, only pixels with ratios equal to or over 2 were considered. MitoTimer and YFP signal colocalization were measured using Mander's algorithm, as previously described (63).

Western blot analysis. Muscle was homogenized in RIPA buffer supplemented with protease and phosphatase A and B (Bimake) inhibitors. Muscle protein carbonyl groups were quantified in quadriceps using the Oxyblot Protein Carbonyl Assay Kit (ab 178020, Abcam) according to the instructions provided by the manufacturer (details are in Supplemental Methods). For all Western blots, protein concentration was measured using the BCA Protein Assay Kit (Thermo Scientific, catalog 23227). Protein lysate was subjected to SDS-PAGE, transferred on nitrocellulose membrane, and blotted using antibodies listed in Supplemental Table 1. Densitometry was performed using Li-COR Image Studio software (Li-COR Biosciences). Full uncut Western blot images are provided in the supplemental material
RNA-Seq and $q R T-P C R$. For transcriptomic profiling, RNA was extracted from quad/gast muscle using TRIzol reagent (Invitrogen), submitted for RNA-Seq, and analyzed informatically as previously described (28). For generating PCA plot, a variance stabilizing transformation function from the DESeq2 (1.30.1) package was applied to the count data. PCA plots were generated using the plotPCA function from the DESeq2 and ggplot2 package in the $\mathrm{R}$ (4.0.3) environment (64). Since no differences were observed between transcript levels of $\mathrm{M}-\mathrm{IR} / \mathrm{IGF}^{\mathrm{f}} \mathrm{R}^{\mathrm{fl} / \mathrm{fl}}(n=3)$ and $\mathrm{M}-\mathrm{Q}^{\mathrm{f} / \mathrm{ll}}(n=2)$ mice, the results in these controls were pooled and compared with those of MIGIRKO and M-QKO mice. All original RNA-Seq data were deposited in the NCBI's Gene Expression Omnibus database (GEO GSE178356). Pairwise $t$ tests were performed on all genes, and FDRs were calculated prior to filtering for OXPHOS genes presented in Figure 7 and Supplemental Table 3. For $\mathrm{T}^{\mathrm{f} / \mathrm{ll}}$ and M-FoxO TKO mice, OXPHOS genes were extracted from a publicly available data set (GEO GSE136948). Transcript levels of STZ diabetic $\mathrm{T}^{\mathrm{f} / \mathrm{fl}}$ mice were compared with those of $\mathrm{T}^{\mathrm{f} / \mathrm{fl}}$ citrate-treated controls, whereas transcripts in M-FoxO TKO and STZ-FoxO TKO were compared with each other and with those of $\mathrm{T}^{\mathrm{fl} / \mathrm{fl}}$ controls.

For qRT-PCR, total mRNA was reverse transcribed to cDNA (Applied Biosystems, catalog 4368814) according to the manufacturer's protocol. RT-PCR was performed using SYBR Green qPCR Master Mix (Bimake or Agilent) with primers for the target genes listed in Supplemental Table 2. Levels of mRNA for genes were normalized either with TATA-box binding protein (TBP) or GAPDH, which were measured on each plate to ensure proper normalization.

$B N-P A G E$. BN-PAGE and in-gel complex I activity were performed as described previously (65), with minor modifications. Briefly, muscle was homogenized in $5 \%$ digitonin, and protein lysate premixed with Coomassie G250 (detergent/Coomassie ratio of 8/8g) was separated on $3 \%$ to $12 \%$ native gradient gels. Electrophoresis was initially conducted at $15 \mathrm{~mA}$ for 15 minutes on ice using anode buffer $(1 \times$ NativePAGE, Thermo Fisher Scientific) and cathode buffer ( $1 \times$ NativePAGE buffer mixed with $0.02 \%$ Coomassie blue dye). For BN-PAGE, cathode buffer was replaced with $0.002 \%$ Coomassie blue containing cathode buffer and gels ran overnight at $2 \mathrm{~mA}$ on ice. After electrophoresis, gels were fixed, destained, and scanned for densitometry. For in-gel activity assays, gels were incubated in complex I assay buffer ( $25 \mathrm{mg}$ of NTB and $100 \mu \mathrm{l}$ of NADH [10 mg/ml] added to $10 \mathrm{ml}$ of 5 $\mathrm{mM}$ Tris/HCl, $\mathrm{pH}$ 7.4) for 5 to 10 minutes. Reaction was stopped with solution containing $50 \%$ methanol and $10 \%$ acetic acid, and gels were scanned for densitometric quantification.

Statistics. All data are presented as mean \pm SEM. Student's 2-tailed $t$ test was performed for comparisons of 2 groups. Two-way ANOVA or 3-way ANOVA was applied for the comparison of 3 or more groups using GraphPad Prism software to determine significance. A $P$ value of less than 0.05 was considered significant for all studies other than RNA-Seq, for which FDR values of less than 0.01 were considered significant for the MIGIRKO and M-QKO experiments. For the STZ FoxO TKO experiments, FDR of less than 0.05 was used to compare STZ to controls. In order to determine whether the transcript levels returned to normal after FoxO1, -3 , and -4 deletion, Student's $t$ test was applied between ratios of STZ-FoxO TKO/M-FoxO TKO and STZ diabetic $\mathrm{T}^{\mathrm{fl} / \mathrm{ll}} /$ control.

Supplemental Methods include full details of experiments.

Study approval. Animal studies were performed according to protocols approved by the IACUC at the University of Iowa. 


\section{Author contributions}

GB designed the study, researched data, and wrote the manuscript. CMP, JJ, PASB, CF, KP, TLJ, AOH, RS, and JDF researched data, helped design experiments, and helped to write the manuscript. PEM and RBS helped in data analysis and in writing the manuscript. VAL, WIS, and EDA provided reagents, helped design experiments, and helped to write the manuscript. BTO designed the study and helped to write the manuscript.

\section{Acknowledgments}

The authors would like to thank the VA Healthcare System for support of this study. This work was supported by VA Merit Review award 101 BXOO4468 (to BTO) and I01 BX000285-05 (to WIS) from the US Department of Veterans Affairs Biomedical Laboratory R\&D (BLRD) Service and by startup funds from Fraternal Order of Eagles Diabetes Research (to BTO and VAL). Postdoc salary of Gourav Bhardwaj was from VA Merit Award
$101 \mathrm{BXOO} 4468$ awarded to BTO. This work was also supported by NIH grants 1R01 HL112413 (to EDA) and 1R01 HL127764 (to EDA), American Heart Association grant 16SDG30360001 (to VAL), and funds from the Iowa Fraternal Order of the Eagles (to WIS). The authors would like to thank C. Ronald Kahn, Jonathan M. Dreyfuss, and Hui Pan from the Joslin Diabetes Center and Harvard Medical School (Boston, Massachusetts, USA) for their assistance in obtaining RNA-Seq data. We thank Eric T. Weatherford (University of Iowa) for help in generation of the PCA plot from RNA-Seq data. We also thank the Fraternal Order of Eagles Diabetes Research Center Metabolic Phenotyping Core Facility for assistance with all the respiration and ATP production data.

Address correspondence to: Brian T. O'Neill, 3314 PBDB, University of Iowa, 169 Newton Road, Iowa City, Iowa 52242, USA. Phone: 319.335.4736; Email: Brian-Oneill@uiowa.edu.
1. Park SW, et al. Accelerated loss of skeletal muscle strength in older adults with type 2 diabetes: the health, aging, and body composition study. Diabetes Care. 2007;30(6):1507-1512.

2. Newman AB, et al. Strength, but not muscle mass, is associated with mortality in the health, aging and body composition study cohort. J Gerontol A Biol Sci Med Sci. 2006;61(1):72-77.

3. Orlando G, et al. The impact of type 1 diabetes and diabetic polyneuropathy on muscle strength and fatigability. Acta Diabetol. 2017;54(6):543-550.

4. Park SW, et al. Decreased muscle strength and quality in older adults with type 2 diabetes: the health, aging, and body composition study. Diabetes. 2006;55(6):1813-1818.

5 . Kelley DE, et al. Dysfunction of mitochondria in human skeletal muscle in type 2 diabetes. Diabetes. 2002;51(10):2944-2950.

6. Mogensen M, et al. Mitochondrial respiration is decreased in skeletal muscle of patients with type 2 diabetes. Diabetes. 2007;56(6):1592-1599.

7. Ruegsegger GN, et al. Altered mitochondrial function in insulin-deficient and insulin-resistant states. JClin Invest. 2018;128(9):3671-3681.

8. Karakelides $\mathrm{H}$, et al. Effect of insulin deprivation on muscle mitochondrial ATP production and gene transcript levels in type 1 diabetic subjects. Diabetes. 2007;56(11):2683-2689.

9. Monaco CMF, et al. Altered mitochondrial bioenergetics and ultrastructure in the skeletal muscle of young adults with type 1 diabetes. Diabetologia. 2018;61(6):1411-1423.

10. De Feyter HM, et al. Early or advanced stage type 2 diabetes is not accompanied by in vivo skeletal muscle mitochondrial dysfunction. Eur J Endocrinol. 2008;158(5):643-653.

11. Fisher-Wellman KH, et al. Mitochondrial respiratory capacity and content are normal in young insulin-resistant obese humans. Diabetes. 2014;63(1):132-141.

12. James HA, et al. Insulin regulation of proteostasis and clinical implications. Cell Metab. 2017;26(2):310-323.

13. Nair KS, et al. Effect of poor diabetic control and obesity on whole body protein metabolism in man. Diabetologia. 1983;25(5):400-403.
14. Meek SE, et al. Differential regulation of amino acid exchange and protein dynamics across splanchnic and skeletal muscle beds by insulin in healthy human subjects. Diabetes. 1998;47(12):1824-1835.

15. Zabielski P, et al. Altered skeletal muscle mitochondrial proteome as the basis of disruption of mitochondrial function in diabetic mice. Diabetes. 2016;65(3):561-573.

16. Yechoor VK, et al. Distinct pathways of insulinregulated versus diabetes-regulated gene expression: an in vivo analysis in MIRKO mice. Proc Natl Acad Sci U S A. 2004;101(47):16525-16530.

17. Schiaffino S, Mammucari C. Regulation of skeletal muscle growth by the IGF1-Akt/PKB pathway: insights from genetic models. Skelet Muscle. 2011;1(1):4.

18. Mavalli MD, et al. Distinct growth hormone receptor signaling modes regulate skeletal muscle development and insulin sensitivity in mice. J Clin Invest. 2010;120(11):4007-4020.

19. O'Neill BT, et al. Differential role of insulin/IGF-1 receptor signaling in muscle growth and glucose homeostasis. Cell Rep. 2015;11(8):1220-1235.

20. O'Neill BT, et al. Insulin and IGF-1 receptors regulate FoxO-mediated signaling in muscle proteostasis. J Clin Invest. 2016;126(9):3433-3446.

21. Eijkelenboom A, Burgering BM. FOXOs: signalling integrators for homeostasis maintenance. Nat Rev Mol Cell Biol. 2013;14(2):83-97.

22. Sanchez AM, et al. FoxO transcription factors: their roles in the maintenance of skeletal muscle homeostasis. Cell Mol Life Sci. 2014;71(9):1657-1671.

23. Hagenbuchner J, Ausserlechner MJ. Mitochondria and FOXO3: breath or die. Front Physiol. 2013;4:147.

24. Kim S, Koh H. Role of FOXO transcription factors in crosstalk between mitochondria and the nucleus. J Bioenerg Biomembr. 2017;49(4):335-341.

25. Ferber EC, et al. FOXO3a regulates reactive oxygen metabolism by inhibiting mitochondrial gene expression. Cell Death Differ. 2012;19(6):968-979.

26. Cheng $\mathrm{Z}$, et al. Foxo1 integrates insulin signaling with mitochondrial function in the liver. Nat Med. 2009;15(11):1307-1311.
27. Romanello V, et al. Mitochondrial fission and remodelling contributes to muscle atrophy. ЕМВО J. 2010;29(10):1774-1785.

28. O'Neill BT, et al. FoxO transcription factors are critical regulators of diabetes-related muscle atrophy. Diabetes. 2019;68(3):556-570.

29. Milan G, et al. Regulation of autophagy and the ubiquitin-proteasome system by the FoxO transcriptional network during muscle atrophy. Nat Commun. 2015;6:6670.

30. Tezze C, et al. Age-associated loss of OPA1 in muscle impacts muscle mass, metabolic homeostasis, systemic inflammation, and epithelial senescence. Cell Metab. 2017;25(6):1374-1389.

31. Murgia M, et al. Single muscle fiber proteomics reveals unexpected mitochondrial specialization. EMBO Rep. 2015;16(3):387-395.

32. Schmidt EK, et al. SUnSET, a nonradioactive method to monitor protein synthesis. Nat Methods. 2009;6(4):275-277.

33. Laplante M, Sabatini DM. mTOR signaling in growth control and disease. Cell. 2012;149(2):274-293.

34. Ju JS, et al. Quantitation of "autophagic flux" in mature skeletal muscle. Autophagy. 2010;6(7):929-935.

35. McWilliams TG, et al. Basal mitophagy occurs independently of PINK1 in mouse tissues of high metabolic demand. Cell Metab. 2018;27(2):439-449.

36. Kubli DA, et al. PINK1 is dispensable for mitochondrial recruitment of parkin and activation of mitophagy in cardiac myocytes. PLoS One. 2015;10(6):e0130707.

37. Wong CY, et al. C2C12 cell model: its role in understanding of insulin resistance at the molecular level and pharmaceutical development at the preclinical stage. JPharm Pharmacol. 2020;72(12):1667-1693.

38. Katayama $\mathrm{H}$, et al. A sensitive and quantitative technique for detecting autophagic events based on lysosomal delivery. Chem Biol. 2011;18(8):1042-1052.

39. Sun N, et al. A fluorescence-based imaging method to measure in vitro and in vivo mitophagy using mt-Keima. Nat Protoc. 2017;12(8):1576-1587. 
40. Laker RC, et al. Ampk phosphorylation of Ulk1 is required for targeting of mitochondria to lysosomes in exercise-induced mitophagy. Nat Commun. 2017;8(1):548.

41. Laker RC, et al. A novel MitoTimer reporter gene for mitochondrial content, structure, stress, and damage in vivo. J Biol Chem. 2014;289(17):12005-12015.

42. Cusi K, et al. Insulin resistance differentially affects the PI 3-kinase- and MAP kinase-mediated signaling in human muscle. JClin Invest. 2000;105(3):311-320.

43. Petersen KF, et al. Impaired mitochondrial activity in the insulin-resistant offspring of patients with type 2 diabetes. N Engl JMed. 2004;350(7):664-671.

44. Patti ME, et al. Coordinated reduction of genes of oxidative metabolism in humans with insulin resistance and diabetes: potential role of PGC1 and NRF1. Proc Natl Acad Sci U S A. 2003;100(14):8466-8471.

45. Bonnard C, et al. Mitochondrial dysfunction results from oxidative stress in the skeletal muscle of diet-induced insulin-resistant mice. JClin Invest. 2008;118(2):789-800.

46. Brunetta HS, et al. Decrement in resting and insulin-stimulated soleus muscle mitochondrial respiration is an early event in diet-induced obesity in mice. Exp Physiol. 2019;104(3):306-321.

47. Franko A, et al. Complete failure of insulin-transmitted signaling, but not obesity-induced insulin resistance, impairs respiratory chain function in muscle. J Mol Med (Berl). 2012;90(10):1145-1160.

48. Toledo FGS, et al. Impact of prolonged overfeeding on skeletal muscle mitochondria in healthy individuals. Diabetologia. 2018;61(2):466-475.

49. Affourtit C. Mitochondrial involvement in skeletal muscle insulin resistance: a case of imbalanced bioenergetics. Biochim Biophys Acta. 2016;1857(10):1678-1693.

50. Li ME, et al. Role of p110a subunit of PI3-kinase in skeletal muscle mitochondrial homeostasis and metabolism. Nat Commun. 2019;10(1):3412.

51. Luo J, et al. Loss of class IA PI3K signaling in muscle leads to impaired muscle growth, insulin response, and hyperlipidemia. Cell Metab. 2006;3(5):355-366.

52. Leermakers PA, Gosker HR. Skeletal muscle mitophagy in chronic disease: implications for muscle oxidative capacity? Curr Opin Clin Nutr Metab Care. 2016;19(6):427-433.

53. Vinothkumar KR, et al. Architecture of mammalian respiratory complex I. Nature. 2014;515(7525):80-84.

54. Webb AE, et al. Characterization of the direct targets of FOXO transcription factors throughout evolution. Aging Cell. 2016;15(4):673-685.

55. Perez-Gomez R, et al. Downregulation of respiratory complex I mediates major signalling changes triggered by TOR activation. Sci Rep. 2020;10(1):4401.

56. Klotz LO, et al. Redox regulation of FoxO transcription factors. Redox Biol. 2015;6:51-72.

57. Fontaine DA, Davis DB. Attention to back- ground strain is essential for metabolic research: C57BL/6 and the international knockout mouse consortium. Diabetes. 2016;65(1):25-33.

58. Ferreira R, et al. Subsarcolemmal and intermyofibrillar mitochondria proteome differences disclose functional specializations in skeletal muscle. Proteomics. 2010;10(17):3142-3154.

59. Schuler M, et al. Temporally controlled targeted somatic mutagenesis in skeletal muscles of the mouse. Genesis. 2005;41(4):165-170.

60. Lark DS, et al. Direct real-time quantification of mitochondrial oxidative phosphorylation efficiency in permeabilized skeletal muscle myofibers. Am J Physiol Cell Physiol. 2016;311(2):C239-C245.

61. Penniman CM, et al. Loss of FoxOs in muscle reveals sex-based differences in insulin sensitivity but mitigates diet-induced obesity. Mol Metab. 2019;30:203-220.

62. Yu L, et al. Mitochondrial function in diabetes: novel methodology and new insight. Diabetes. 2013;62(6):1833-1842.

63. Bravo R, et al. Increased ER-mitochondrial coupling promotes mitochondrial respiration and bioenergetics during early phases of ER stress. J Cell Sci. 2011;124(pt 13):2143-2152.

64. Love MI, et al. Moderated estimation of fold change and dispersion for RNA-Seq data with DESeq2. Genome Biol. 2014;15(12):550.

65. Wittig I, et al. High resolution clear native electrophoresis for in-gel functional assays and fluorescence studies of membrane protein complexes. Mol Cell Proteomics. 2007;6(7):1215-1225. 